\title{
Optimal Expected Exponential Utility of Dividend Payments in a Brownian Risk Model
}

\author{
P. Grandits* \\ F. Hubalek* \\ W. Schachermayer* \\ M. Žigo ${ }^{\dagger}$
}

November 17, 2006

\begin{abstract}
We consider the following optimization problem for an insurance company

$$
\max _{C} \mathrm{E}\left[U\left(\int_{0}^{\infty} e^{-\beta t} d C_{t}\right)\right]
$$

Here $U(x)=(1-\exp (-\gamma x)) / \gamma$ denotes an exponential utility function with risk aversion parameter $\gamma, C$ denotes the accumulated dividend process, and $\beta$ a discount factor. We show that - assuming that a certain integral equation has a solution - the optimal strategy is a barrier strategy. The barrier function is a solution of the integral equation and turns out to be time-dependent.

In addition we study the problem from a different point of view, namely by using a certain ansatz for the value function and the barrier.
\end{abstract}

Keywords: optimal dividend payment, optimal control, free boundary value problem

\section{Introduction}

Insurance companies face a certain dilemma between choosing a strategy maximizing their dividends (in one way or the other) and a more conservative approach, which can be expressed e.g., by demanding a low probability of ruin. The literature on both topics is vast. We mention just a few of the articles. In Gaier, Grandits, and Schachermayer (2003), Hipp and Plum (2000), Schmidli (2005), Paulsen (2002), Kalashnikov and Norberg (2002) or Grandits (2004), insurance companies are considered, which invest in the stock market (or face a stochastic interest rate on their reserves). The problem is to find estimates for the ruin probability, or to find an investment strategy, which minimizes this probability.

As regards the maximization of dividends several approaches are possible. On the one hand side one can distinguish between the ways one models the reserve of the company, e.g. diffusion approximation, compound Poisson process or a general renewal model are used. On the other hand one has the possibility to fix the strategy (e.g. a linear dividend barrier as in Gerber (1981) or a nonlinear one as in Albrecher and Kainhofer (2002)) and to try to estimate the expected discounted dividends by analytical or numerical methods). Another approach is to try to find the optimal strategy in a model, which is simple enough. E.g. the article by Gerber (1969) for the compound Poisson case and Asmussen and Taksar (1997) for the diffusion approximation

\footnotetext{
*Institute for Mathematical Methods in Economics, TU Vienna, 1040 Vienna, Austria

${ }^{\dagger}$ Department of Mathematics, University Zagreb, 10000 Zagreb, Croatia
} 
case (see also the paper Shreve et al. (1984) for a more general diffusion process than Brownian motion with drift). In the diffusion case it turns out that a strategy with a constant barrier is optimal, i.e. one pays out everything as dividends which is above a certain level. In the compound Poisson case the strategy is in general a more complicated so called "band strategy" (see Gerber (1969) for details).

Another approach to maximize dividends is by using utility functions. E.g. in Hubalek and Schachermayer (2004) the following problem is considered

$$
\max _{c} \mathrm{E}\left[\int_{0}^{\tau} e^{-\beta t} U\left(c_{t}\right) d t\right]
$$

Here $U(x)$ is a utility function, $\beta$ a discounting factor, $c$ a dividend rate and $\tau$ the time of ruin. The economic interpretation of the target functional is the following: At each instant of time one calculates the "utility rate" $U(c(t))$ of the paid dividend $c(t)$. Finally one calculates the discounted "sum" of this rate until ruin occurs.

The problem we want to consider in the present paper is only formally similar to this. We just move the utility function - we will always use exponential utility - outside the integral and use the integrated form of the dividend payment stream $\left(C_{t}=\int_{0}^{t} c_{s} d s\right)$

$$
\max _{C} \mathrm{E}\left[U\left(\int_{0}^{\tau} e^{-\beta t} d C_{t}\right)\right] .
$$

The economic interpretation is now of course completely different. Here we want to maximize the expected utility of the present value of dividends until ruin. This problem was suggested in Gerber and Shiu (2004). We finally mention that we use a diffusion approximation, or more precisely, Brownian motion with drift for the surplus process.

The problem (2) is not only different from the economic interpretation, but also from the mathematical point of view. It turns out that it is a singular optimal control problem, see Fleming and Soner (1993), chapter VIII. This means that the optimal solution $C^{*}$ is not absolutely continuous with respect to Lebesgue measure. Is is rather of so called "barrier type", meaning that it is optimal for the company to pay out everything which is above a certain level, which is described by a barrier function. In our case this barrier function is time-dependent.

One should also remark that it is not possible to formulate our problem as a one-dimensional problem, i.e. to use only one state variable namely the surplus process of the company, say $X_{t}$. One is forced to introduced a second one, namely the discounted dividends until time $t$, say $Y_{t}$. This gives, using Hamilton-Jacobi-Bellman techniques, a three (the optimal strategy is not independent of time) dimensional free boundary value problem for the value function

$$
W(t, x, y)=\sup _{C} \mathrm{E}\left[U\left(\int_{0}^{\tau} e^{-\beta t} d C_{t}\right) \mid X_{t}=x, Y_{t}=y\right] .
$$

Usage of the exponential utility function $U(x)=\left(1-e^{-\gamma x}\right) / \gamma$ reduces the problem to a twodimensional one. We are not able to solve this free boundary problem completely rigorously, but we were able to derive a certain integral equation for the barrier function. Under the assumption that this equation has a $C^{1}$-solution $b(t)$, we can prove that $b(t)$ is the barrier we are looking for. In Section 1.4 we try to solve the free boundary value problem by using a certain series ansatz for the boundary function and the value function. We obtain recursions for the coefficients of the series, and numerical experiments indicate, that our series produce meaningful results, but we have been unable to prove convergence. 


\section{The model and the main result}

\subsection{The model}

The wealth of an insurance company is modeled by a Brownian motion with drift (see e.g. Asmussen and Taksar (1997) for a motivation of this choice), i.e.,

$$
R_{t}=x+\mu t+\sigma W_{t}, \quad t \geq 0,
$$

where $x>0$ denotes the initial reserve, $\mu \in \mathbb{R}$ the drift parameter and $\sigma>0$ the diffusion parameter. $W$ is a standard Brownian motion defined on (and adapted to) some filtered probability space $(\Omega, \mathcal{F}, P, \mathbb{F})$ with $\mathbb{F}=\left(\mathcal{F}_{t}\right)_{t \geq 0}$. A dividend paying strategy consists in an adapted increasing right-continuous process $\left(C_{t}\right)_{t \geq 0}$, modeling the accumulated dividend payments up to time $t$. Define the ex-dividend process $X$ by letting

$$
X_{t}=R_{t}-C_{t}, \quad t \geq 0
$$

and the time of ruin under the dividend paying policy $C$ by

$$
\tau=\inf \left\{t: X_{t} \leq 0\right\} .
$$

A dividend policy is admissible if it is right-continuous, non-decreasing process $C_{t}$, such that $X_{t} \geq 0$ for all $t \leq \tau$ and $C_{t}=C_{\tau}$ for $t \geq \tau$, as well as $C_{0-}=0$. This means that after the moment of ruin no dividend payments are possible any more and the amount of dividends one pays out at a certain instant $t$ is bounded above by the ex-dividend process $X_{t}$.

We want to maximize expected exponential utility of discounted dividend payments, i.e. we look for an admissible dividend paying policy $C^{*}$ such that

$$
\mathrm{E}\left[U\left(\int_{0}^{\infty} e^{-\beta t} d C_{t}^{*}\right)\right]=\max _{C} \mathrm{E}\left[U\left(\int_{0}^{\infty} e^{-\beta t} d C_{t}\right)\right] .
$$

Here $\beta>0$ denotes a fixed discount rate, and the utility function $U$ is given by

$$
U(x)=\frac{1}{\gamma}\left(1-e^{-\gamma x}\right)
$$

where $\gamma>0$ is also fixed.

The limiting case $\gamma \rightarrow 0$ (no risk aversion !), i.e. $U(x)=x$, was already studied by Asmussen and Taksar (1997). They found that, in their case the optimal dividend paying strategy is of barrier type with time independent barrier function. It consists in paying out all the reserve exceeding a certain constant level $b_{0}$ as dividends. The level $b_{0}$ is given by

$$
b_{0}=\frac{1}{\lambda_{1}-\lambda_{2}} \ln \left(\frac{\lambda_{2}^{2}}{\lambda_{1}^{2}}\right)
$$

where $\lambda_{1}$ and $\lambda_{2}$ are given by

$$
\lambda_{1}=\frac{-\mu+\sqrt{\mu^{2}+2 \beta \sigma^{2}}}{\sigma^{2}}>0, \quad \lambda_{2}=\frac{-\mu-\sqrt{\mu^{2}+2 \beta \sigma^{2}}}{\sigma^{2}}<0 .
$$

We will prove that in the case of exponential utility (7), the optimal dividend paying policy is also of barrier type. This barrier $b(t)$ is time-dependent and it satisfies

$$
\lim _{t \rightarrow \infty} b(t)=b_{0}
$$

where $b_{0}$ is as above. 


\subsection{The basic idea}

To avoid technicalities we consider in the following discussion the case when the dividend policy $\left(C_{t}\right)_{t \geq 0}$ admits a density $\left(c_{s}\right)_{s \geq 0} \geq 0$ (knowing very well that eventually this will not be the case for the optimizer). In differential form, the model is now given by

$$
\begin{aligned}
d X_{t} & =\left(\mu-c_{t}\right) d t+\sigma d W_{t}, \quad X_{0}=x, \\
d Y_{t} & =e^{-\beta t} c_{t} d t, \quad Y_{0}=0 .
\end{aligned}
$$

We define the value function

$$
W(t, x, y)=\sup _{c} E\left[U\left(\int_{t}^{+\infty} e^{-\beta s} c_{s} d s+y\right) \mid X_{t}=x, Y_{t}=y\right] .
$$

We can now write

$$
W(t, x, y)=U(y)+w(t, x, y),
$$

where $w(t, x, y)$ denotes the optimal additional expected utility, conditionally on $X_{t}=x, Y_{t}=y$.

It is a fundamental paradigm known in the theory of stochastic control (see e.g. Fleming and Soner (1993)), that - under appropriate regularity assumptions - for the optimal process $\left(X_{t}^{*}, Y_{t}^{*}\right)$, the process $W\left(t, X_{t}^{*}, Y_{t}^{*}\right)$ must be a martingale. Using Itô's formula and setting the drift term to be equal to zero we obtain

$$
w_{t}+\mu w_{x}+\frac{\sigma^{2}}{2} w_{x x}+\sup _{c \geq 0}\left[c\left(-w_{x}+e^{-\beta t} w_{y}+e^{-\beta t} U^{\prime}(y)\right)\right]=0 .
$$

The function $w$ has an obvious scaling property

$$
w(t, x, y)=e^{-\gamma y} w(t, x, 0),
$$

which reduces the 3 -dimensional problem to a 2 -dimensional one. We therefore set $w(t, x):=$ $w(t, x, 0)$, and (14) becomes

$$
w_{t}+\mu w_{x}+\frac{\sigma^{2}}{2} w_{x x}+\sup _{c \geq 0}\left[c\left(-w_{x}+e^{-\beta t}(1-\gamma w)\right)\right]=0 .
$$

In order for the problem to make sense, we obviously must have the inequalities

$$
\begin{array}{r}
w_{x} \geq e^{-\beta t}(1-\gamma w) \\
w_{t}+\mu w_{x}+\frac{\sigma^{2}}{2} w_{x x} \leq 0
\end{array}
$$

When the first inequality above is strict, clearly the optimal dividend strategy is $c_{t}^{*}=0$ and we also have $w_{t}+\mu w_{x}+\frac{\sigma^{2}}{2} w_{x x}=0$. If the second inequality is strict the only chance to satisfy (16) is to choose " $c^{*}=\infty$ ". Note that we are here only at an informal level, and we will find in the proof of our main theorem that this has to be interpreted as paying out a certain amount of dividend at once. We can combine all this to

$$
\max \left\{w_{t}+\mu w_{x}+\frac{\sigma^{2}}{2} w_{x x},-w_{x}+e^{-\beta t}(1-\gamma w)\right\}=0
$$

The only situation where we pay out any dividends is when the first inequality in (17) becomes an equality. Hence we expect that the optimal strategy is of barrier type. In other words, we expect that there is a barrier function $x=b(t)$ such that 


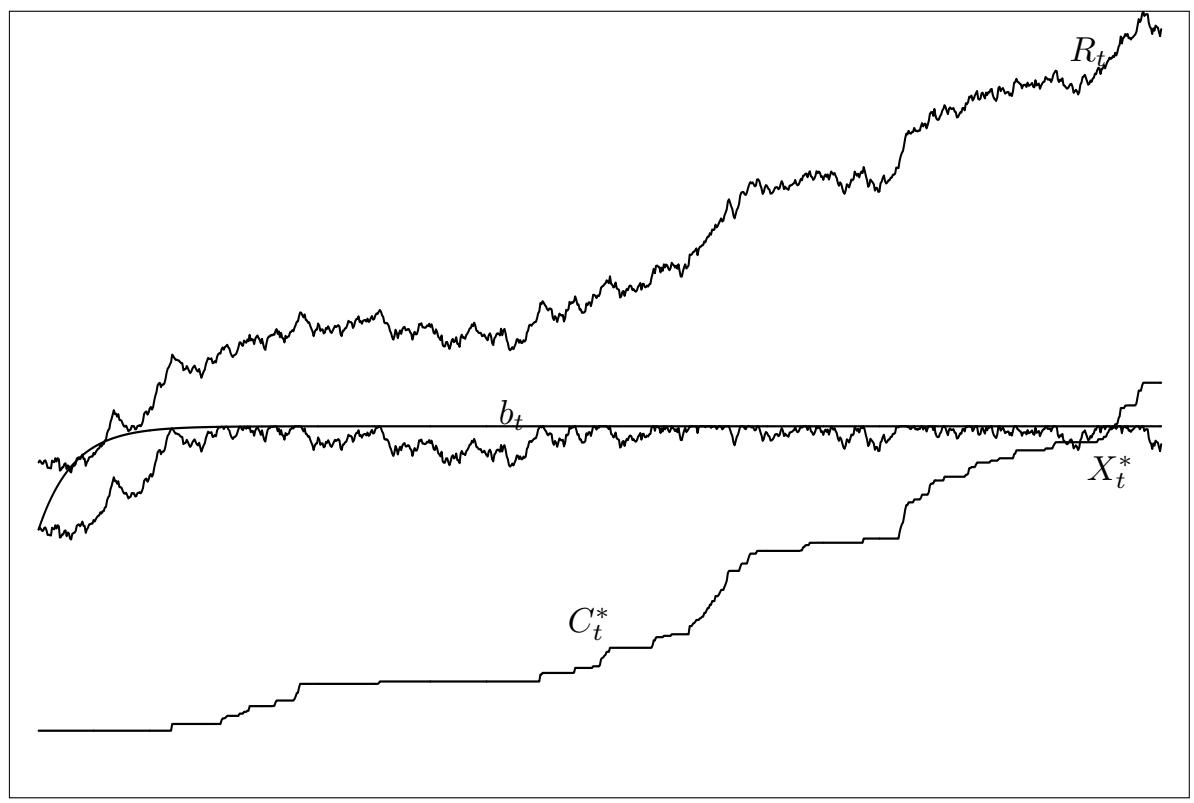

Figure 1: Reserve, ex-dividend and dividend processes.

- for $x \leq b(t)$ we do not pay out any dividends

- for $x>b(t)$ we pay out everything above reserve $b(t)$.

See Figure 1. Such a barrier function divides $[0,+\infty) \times \mathbb{R}_{+}$into two regions, so we denote

$$
\begin{aligned}
I & =\left\{(t, x) \in \mathbb{R}_{+}^{2} ; t \geq 0, x \leq b(t)\right\} \\
I I & =\left\{(t, x) \in \mathbb{R}_{+}^{2} ; t \geq 0, x>b(t)\right\} .
\end{aligned}
$$

Reformulating our conclusions in terms of function $w$, we expect that there is a boundary function $x=b(t)$ such that following conditions hold true:

$$
\begin{gathered}
w_{t}+\mu w_{x}+\frac{\sigma^{2}}{2} w_{x x}=0, \\
w_{x} \geq e^{-\beta t}(1-\gamma w), \quad(t, x) \in I, \\
\text { and } \begin{array}{c}
w_{t}+\mu w_{x}+\frac{\sigma^{2}}{2} w_{x x} \leq 0, \\
w_{x}=e^{-\beta t}(1-\gamma w), \quad(t, x) \in I I .
\end{array}
\end{gathered}
$$

Denote by $w^{I}$ the solution to the $\mathrm{PDE} w_{t}+\mu w_{x}+\frac{\sigma^{2}}{2} w_{x x}=0$ in region $\mathrm{I}$, and by $w^{I I}$ the solution to the ODE $w_{x}=e^{-\beta t}(1-\gamma w)$ in region II. We expect that our optimal function $w$ is a combination of these two. The general form of the solution of the ODE is given by

$$
w^{I I}(t, x)=U\left(e^{-\beta t}(x+d(t))\right)
$$

where $d(t)$ is some integration constant, possibly depending on $t$. For determining $d(t)$ and for the missing boundary data of $w^{I}$, we use the principle of smooth fit in the following way: We 
ask the functions $w, w_{x}$, and $w_{t}+\mu w_{x}+\frac{\sigma^{2}}{2} w_{x x}=: \mathcal{L} w$ to be continuous over the boundary (the last condition is equivalent to demanding continuity of $w_{x x}$, but it is more convenient).

The computational details of the following arguments can be found in the proof of Proposition 2. The following should motivate the appearance of our basic integral equation for the boundary function $b(t)$. The existence of a solution of this integral equation will show up in the standing assumption of this paper, which we shall formulate below.

The smooth fit condition above gives

$$
\mathcal{L}\left[U\left(e^{-\beta t}(x+d(t))\right)\right]_{\mid x=b(t)}=0
$$

Hence $d(t)=d[b(s), s \geq t]$, which means that $d(t)$ can be expressed as a functional of the future of $b$. This gives us

$$
D(t):=w^{I I}(t, b(t))=D[b(s), s \geq t] .
$$

Since we want also continuity of $w$ over the boundary we use this $D(t)$ as boundary value for the following BVP

$$
\begin{aligned}
w_{t}+\mu w_{x}+\frac{\sigma^{2}}{2} w_{x x} & =0, \\
w(t=\infty, x) & =0, \\
w(t, x=0) & =0, \\
w(t, b(t)) & =D(t) .
\end{aligned}
$$

This gives us a solution $w^{I}$ in domain $I$, depending on the future of $b(t)$. Finally we use Green's functions to express $w_{x}^{I}$ at the boundary as functional of the future of $b(t)$. Equating this to $w_{x}^{I I}$, which is explicitly known, yields our fundamental integral equation, which shows up in the following

\section{Standing assumption:}

The following equation

$$
\int_{0}^{\infty} \int_{0}^{b(r+t)} G(r, x, b(t)) g(r+t, x) d x d r=\frac{\sigma^{2}}{2} \int_{0}^{\infty} G(r, b(r+t), b(t)) \tilde{u}(r+t) d r,
$$

has a positive solution $b(t)_{t \geq 0}$, which fulfills: $b(t) \in C^{1}\left(\mathbb{R}^{+}\right)$with $\lim _{t \rightarrow \infty} b(t)=b(\infty)>0$ and $b^{\prime}(t)=\mathcal{O}\left(e^{-\frac{\beta}{2} t}\right)$.

Here $G(r, x, y)$ denotes a certain Green's function (to be described in the proof of Proposition 2, eq. (128) of the operator

$$
L^{*} u=-u_{t}+\frac{\sigma^{2}}{2} u_{x x}
$$

and $g$ as well as $\tilde{u}$ are functionals of the future of $b(t)$, also described there in equations (123) and (142).

Remark. We close this section with a certain scaling property of the function $w$. Write now $w^{(\gamma)}$ for $w$ to indicate the dependence of the solution on the parameter $\gamma$. Then we have for $t_{0}>0$

$$
w^{(\gamma)}\left(t+t_{0}, x\right)=e^{-\beta t_{0}} w^{\left(\gamma e^{\left.-\beta t_{0}\right)}\right.}(t, x)
$$

and in particular

$$
w^{(\gamma)}\left(t_{0}, x\right)=e^{-\beta t_{0}} w^{\left(\gamma e^{-\beta t_{0}}\right)}(0, x)
$$


We show (28). To find $w^{(\gamma)}\left(t_{0}, x\right)$ we have to find the optimal process $\left(C_{t}^{*}\right)_{t \geq t_{0}}$ of accumulated dividends conditionally on $X_{t_{0}}=x$ so that

$$
w^{(\gamma)}\left(t_{0}, x\right)=\mathrm{E}\left[\frac{1-\exp \left(-\gamma \int_{t_{0}}^{\infty} e^{-\beta t} d C_{t}^{*}\right)}{\gamma}\right]
$$

Hence

$$
w^{(\gamma)}\left(t_{0}, x\right)=e^{-\beta t_{0}} \mathrm{E}\left[\frac{1-\exp \left(-\gamma e^{-\beta t_{0}} \int_{0}^{\infty} e^{-\beta s} d C_{s+t_{0}}^{*}\right)}{\gamma e^{-\beta t_{0}}}\right]=e^{-\beta t_{0}} w^{\left(\gamma e^{-\beta t_{0}}\right)}(0, x)
$$

and $\left(C_{s+t_{0}}^{*}\right)_{s \geq 0}$ is clearly optimal for $U^{\left(\gamma e^{-\beta t_{0}}\right)}$, conditionally on $X_{0}=x$ if $\left(C_{t}\right)_{t \geq t_{0}}$ was optimal in the previous setting. This shows (28) and (27) follows by the same token.

\subsection{The main result}

We start with recalling our model:

$$
\begin{aligned}
d X_{t}= & \mu d t+\sigma d W_{t}-d C_{t}, \quad X_{0-}=x \\
d Y_{t}= & e^{-\beta t} d C_{t}, \quad Y_{0-}=0, \\
& \mathrm{E}\left[U\left(\int_{0}^{\infty} e^{-\beta t} d C_{t}\right)\right] \mapsto \max ,
\end{aligned}
$$

where $\left(C_{t}\right)_{t \geq 0}$ has to be admissible and we do not assume that it has a density any more. Now we are ready to state the main result.

Theorem 1 (The main theorem) If $\gamma \geq \frac{2 \mu}{\sigma^{2}}$, the solution of (29) is to pay out immediately the whole endowment, i.e. $C_{t}=x$ for $t \geq 0$ and $C_{0-}=0$.

Under our standing assumptions and if $\gamma<\frac{2 \mu}{\sigma^{2}}$, the barrier strategy with barrier $b(t)$, where $b(t)$ solves (25) is the optimal strategy.

In order to prove this theorem we shall need several auxiliary results, which we state now, but prove only later on.

Proposition 1 Given a strictly positive $C^{1}$ boundary $b(t)$ with $\lim _{t \rightarrow \infty} b(t)=b(\infty)>0$ and $b^{\prime}(t)=\mathcal{O}\left(e^{-\frac{\beta}{2} t}\right)$, and given a function $D(t) \in C^{1}$ with

$$
-D^{\prime}(t) \sim \text { const. } e^{-\beta t} \sim \text { const. } D(t),
$$

where the constants are not necessarily the same positive constants depending on the model parameters, consider the following boundary value problem

$$
\begin{aligned}
w_{t}+\mu w_{x}+\frac{\sigma^{2}}{2} w_{x x} & =0, \\
w(t=\infty, x) & =0, \\
w(t, x=0) & =0, \\
w(t, b(t)) & =D(t) .
\end{aligned}
$$

Then we have: The PDE in (31) has a solution which fulfills

$$
\begin{aligned}
w_{x} & \in C(M), \\
w_{t} & \in C(M), \\
w_{x x} & \in C(M) .
\end{aligned}
$$


where $M=\{(t, x) \mid t \geq 0,0 \leq x \leq b(t)\}$. Note that at points $t_{0}$, where $b^{\prime}\left(t_{0}\right)=0$, we possibly can not define $w_{t}$ up to the boundary. In this case we take $\lim _{x / b\left(t_{0}\right)} w_{t}\left(x, t_{0}\right)$.

We also have

$$
\left\|w_{x}(t, x)\right\|_{C[0, b(t)]}=\mathcal{O}\left(e^{-\frac{t}{N}}\right),
$$

for some $N>0$.

Proposition 2 Assume that there exists a strictly positive $C^{1}\left(\mathbb{R}_{+}\right)$boundary $b(t)$, which solves the basic integral equation (25) of our standing assumption, and which divides $\mathbb{R}_{+}^{2}$ into regions $I=\left\{(t, x) \in \mathbb{R}_{+}^{2} ; t \geq 0, x \leq b(t)\right\}$ and $I I=\left\{(t, x) \in \mathbb{R}_{+}^{2} ; t \geq 0, x>b(t)\right\}$. Then there exists a function $w(t, x)=w^{I}(t, x) \cdot 1_{I}(t, x)+w^{I I}(t, x) \cdot 1_{I I}(t, x)$ such that

(i) $w_{t}^{I}+\mu w_{x}^{I}+\frac{\sigma^{2}}{2} w_{x x}^{I}=0$ in $I$,

(ii) $w_{x}^{I I}=e^{-\beta t}\left(1-\gamma w^{I I}\right)$ in $I I$,

(iii) $w(t, 0)=0$ for $t>T$,

(iv) $w$ is bounded,

(v) $w^{I}=w^{I I}$ at the boundary $(t, b(t))$,

(vi) $w_{x}^{I}=w_{x}^{I I}$ at the boundary $(t, b(t))$,

(vii) $w_{t}+\mu w_{x}+\frac{\sigma^{2}}{2} w_{x x}=0$ at the boundary $(t, b(t))$.

We also have

(viii) $w_{t}$ and $w_{x x}$ exist and are continuous over the boundary.

Proposition 3 If $\gamma<\frac{2 \mu}{\sigma^{2}}$ the solution $w$ of proposition 2 satisfies

(i) $w_{t}^{I I}+\mu w_{x}^{I I}+\frac{\sigma^{2}}{2} w_{x x}^{I I} \leq 0$ in $I I$,

(ii) $w_{x}^{I} \geq e^{-\beta t}\left(1-\gamma w^{I}\right)$ in $I$.

We relegate the proofs of propositions 1, 2 and 3 to sections 2,3 and 4. Assuming the validity of these propositions we give now the proof of our main theorem.

Proof of Theorem 1. We start with

Case 1: $\gamma \geq \frac{2 \mu}{\sigma^{2}}$ :

Clearly the expected utility of dividend payments, using the strategy suggested in the theorem is given by

$$
\frac{1-e^{-\gamma x}}{\gamma}=U(x) \text {. }
$$

On the other hand we have $S_{t} \equiv x+\mu t+\sigma W_{t} \geq C_{t}$ for all $t \leq \tau$, and all admissible strategies $C_{t}$. Hence

$$
\begin{aligned}
& \sup _{C} \mathrm{E}\left[U\left(\int_{0}^{\tau} e^{-\beta t} d C_{t}\right)\right] \leq \sup _{C} \mathrm{E}\left[U\left(C_{\tau}\right)\right] \leq \\
& \sup _{C} \mathrm{E}\left[U\left(S_{\tau}\right)\right]=\mathrm{E}\left[U\left(S_{\tau}\right)\right] \leq U\left(S_{0}\right)=U(x),
\end{aligned}
$$

where the last inequality holds, since for $\gamma \geq \frac{2 \mu}{\sigma^{2}}$ the process $U\left(S_{t \wedge \tau}\right)$ is a bounded supermartingal. This concludes the proof of the first case. 
Now we consider

Case 2: $\gamma<\frac{2 \mu}{\sigma^{2}}$ :

We divide the proof into the following two lemmas. Let $V$ be defined by

$$
V(t, x, y)=U(y)+e^{-\gamma y} w(t, x), \quad t \geq 0 ; x, y \geq 0 .
$$

Lemma 1 Let $b$ and $w$ be as in proposition 2. For any admissible strategy $\left(C_{t}\right)_{t \geq 0}$, we have that the process

$$
V\left(t, X_{t \wedge \tau}, Y_{t \wedge \tau}\right)=U\left(Y_{t \wedge \tau}\right)+e^{-\gamma Y_{t \wedge \tau}} w\left(t, X_{t \wedge \tau}\right), \quad t \geq 0
$$

is a nonnegative supermartingale. It follows

$$
V(0, x, 0)=w(0, x) \geq E\left[U\left(\int_{0}^{\tau} e^{-\beta s} d C_{s}\right)\right]=E\left[U\left(\int_{0}^{\tau} e^{-\beta s} d C_{s}\right)\right] .
$$

Proof. It follows from the proposition above that the process $w\left(t, X_{t}\right)$ is a semimartingale, so we can write

$$
\begin{aligned}
d V\left(t, X_{t}, Y_{t}\right)= & d U\left(Y_{t}\right)+d\left(e^{-\gamma Y_{t}} w\left(t, X_{t}\right)\right) \\
= & d U\left(Y_{t}\right)+e^{-\gamma Y_{t-}} d\left(w\left(t, X_{t}\right)\right)+w\left(t, X_{t-}\right) d\left(e^{-\gamma Y_{t}}\right) \\
& +d\left[e^{-\gamma Y_{t}}, w\left(t, X_{t}\right)\right]
\end{aligned}
$$

Since $\left(Y_{t}\right)$ is of finite variation, we have (see Protter (1995), theorem 31)

$$
\begin{aligned}
d U\left(Y_{t}\right) & =U^{\prime}\left(Y_{t-}\right) d Y_{t}+U\left(Y_{t}\right)-U\left(Y_{t-}\right)-U^{\prime}\left(Y_{t-}\right) \triangle Y_{t} \\
& =e^{-\gamma Y_{t-}} e^{-\beta t} d C_{t}+U\left(Y_{t}\right)-U\left(Y_{t-}\right)-U^{\prime}\left(Y_{t-}\right) \triangle Y_{t} .
\end{aligned}
$$

Now we use $U^{\prime}(y)=e^{-\gamma y}$ and $\triangle Y_{t}=e^{-\beta t} \triangle C_{t}$. When the integrator is continuous, we can pass from left limits to the value at $t$ in the integrand. This gives us

$$
\begin{aligned}
d U\left(Y_{t}\right) & =e^{-\gamma Y_{t-}} e^{-\beta t} d C_{t}+\frac{1}{\gamma}\left(e^{-\gamma Y_{t-}}-e^{-\gamma Y_{t}}\right)-e^{-\gamma Y_{t-}} e^{-\beta t} \Delta C_{t} \\
& =e^{-\gamma Y_{t}} e^{-\beta t} d C_{t}^{c}+\frac{1}{\gamma}\left(e^{-\gamma Y_{t-}}-e^{-\gamma Y_{t}}\right),
\end{aligned}
$$

where $\left(C_{t}^{c}\right)$ denotes continuous part of the process $\left(C_{t}\right)$. It follows

$$
d\left(e^{-\gamma Y_{t}}\right)=-\gamma d U\left(Y_{t}\right)=-\gamma e^{-\gamma Y_{t}} e^{-\beta t} d C_{t}^{c}+\left(e^{-\gamma Y_{t}}-e^{-\gamma Y_{t-}}\right) .
$$

Furthermore, since $\left(e^{-\gamma Y_{t}}\right)$ is of finite variation it follows from theorems 26 and 28 of Protter (1995) that

$$
d\left[e^{-\gamma Y_{t}}, w\left(t, X_{t}\right)\right]_{t}=\left(e^{-\gamma Y_{t}}-e^{-\gamma Y_{t-}}\right)\left(w\left(t, X_{t}\right)-w\left(t, X_{t-}\right)\right) .
$$

By Ito's lemma we have

$$
\begin{aligned}
d w\left(t, X_{t}\right)= & w_{t}\left(t, X_{t}\right) d t+w_{x}\left(t, X_{t-}\right) d X_{t}+\frac{\sigma^{2}}{2} w_{x x}\left(t, X_{t-}\right) d[X, X]_{t}^{c} \\
& +w\left(t, X_{t}\right)-w\left(t, X_{t-}\right)-w_{x}\left(t, X_{t-}\right) \triangle X_{t} \\
= & \left(w_{t}\left(t, X_{t}\right)+\mu w_{x}\left(t, X_{t}\right)+\frac{\sigma^{2}}{2} w_{x x}\left(t, X_{t}\right)\right) d t+\sigma w_{x}\left(t, X_{t}\right) d W_{t} \\
& -w_{x}\left(t, X_{t}\right) d C_{t}^{c}+w\left(t, X_{t}\right)-w\left(t, X_{t-}\right) .
\end{aligned}
$$


Plugging (40), (41), (42) and (43) into (39), after some cancellations we get

$$
\begin{aligned}
d V\left(t, X_{t}, Y_{t}\right)= & e^{-\gamma Y_{t}}\left(w_{t}\left(t, X_{t}\right)+\mu w_{x}\left(t, X_{t}\right)+\frac{\sigma^{2}}{2} w_{x x}\left(t, X_{t}\right)\right) d t \\
& +\sigma e^{-\gamma Y_{t}} w_{x}\left(t, X_{t}\right) d W_{t} \\
& +e^{-\gamma Y_{t}}\left(-w_{x}\left(t, X_{t}\right)+e^{-\beta t}\left(1-\gamma w_{x}\left(t, X_{t}\right)\right)\right) d C_{t}^{c} \\
& +\frac{1}{\gamma}\left(e^{-\gamma Y_{t-}}-e^{-\gamma Y_{t}}\right)+e^{-\gamma Y_{t}} w\left(t, X_{t}\right)-e^{-\gamma Y_{t-}} w\left(t, X_{t-}\right) .
\end{aligned}
$$

The jump part of (44) is equal to $V\left(t, X_{t}, Y_{t}\right)-V\left(t, X_{t-}, Y_{t-}\right)$. Passing to the integral form of (44) gives

$$
\begin{aligned}
V\left(t, X_{t \wedge \tau}, Y_{t \wedge \tau}\right)= & V(0, x, 0) \\
& +\int_{0}^{t \wedge \tau} e^{-\gamma Y_{s}}\left(w_{t}\left(s, X_{s}\right)+\mu w_{x}\left(s, X_{s}\right)+\frac{\sigma^{2}}{2} w_{x x}\left(s, X_{s}\right)\right) d t \\
& +\sigma \int_{0}^{t \wedge \tau} e^{-\gamma Y_{s}} w_{x}\left(s, X_{s}\right) d W_{s} \\
& +\int_{0}^{t \wedge \tau} e^{-\gamma Y_{s}}\left(-w_{x}\left(s, X_{s}\right)+e^{-\beta s}\left(1-\gamma w_{x}\left(s, X_{s}\right)\right)\right) d C_{s}^{c} \\
& +\sum_{0 \leq s \leq t \wedge \tau}\left(V\left(s, X_{s}, Y_{s}\right)-V\left(s, X_{s-}, Y_{s-}\right)\right) .
\end{aligned}
$$

Next we note that for every $s \geq 0$ we have

$$
V\left(s, X_{s}, Y_{s}\right)-V\left(s, X_{s-}, Y_{s-}\right) \leq 0 .
$$

Indeed, by the mean value theorem there is a point $(s, \theta, \vartheta)$ between $\left(s, X_{s}, Y_{s}\right)$ and $\left(s, X_{s-}, Y_{s-}\right)$ such that

$$
\begin{aligned}
V\left(s, X_{s}, Y_{s}\right)-V\left(s, X_{s-}, Y_{s-}\right) & =V_{x}(s, \theta, \vartheta) \triangle X_{s}+V_{y}(s, \theta, \vartheta) \triangle Y_{s} \\
& =e^{-\gamma \vartheta}\left(e^{-\beta s}(1-\gamma w(s, \theta))-w_{x}(s, \theta)\right) \triangle C_{s} \\
& \leq 0,
\end{aligned}
$$

where we have used proposition 3 and proposition 2 (for region $I$ ) in the last step. Taking expectation in (45), by proposition 2 and proposition 3

$$
V(0, x, 0)=w(0, x) \geq \mathrm{E}\left[V\left(t, X_{t \wedge \tau}, Y_{t \wedge \tau}\right)\right] \geq \mathrm{E}\left[U\left(Y_{t \wedge \tau}\right)\right],
$$

so the result follows by letting $t \rightarrow \infty$ and using dominated convergence theorem.

Our next step is to construct a process $C^{*}$ such that

$$
V(0, x, 0)=w(0, x)=E\left[U\left(\int_{0}^{\tau} e^{-\beta s} d C_{s}^{*}\right)\right] .
$$

Let $w$ and $b$ be as in proposition 2 and define

$$
\begin{aligned}
C_{t}^{*} & =\max _{0 \leq s \leq t}\left[x+\mu s+\sigma W_{s}-b(s)\right]_{+}, \\
X_{t}^{*} & =x+\mu t+\sigma W_{t}-C_{t}^{*} .
\end{aligned}
$$


$C_{t}^{*}$ is an increasing process for which $C_{0}>0$ iff $x>b(0)\left(C_{0-}=0\right)$. Moreover, $C_{t}^{*}$ increases only at times when $X^{*}(t)=b(t)$ (see Figure 1). More precisely, we have

$$
\begin{aligned}
& X_{t}^{*} \leq b(t), \\
& \int_{0}^{+\infty} 1_{\left\{X_{t}^{*}<b(t)\right\}} d C_{t}^{*}=0 .
\end{aligned}
$$

Lemma 2 For $C_{t}^{*}$ and $X_{t}^{*}$ defined by (49) equation (48) holds.

Since $X_{t}^{*}, C_{t}^{*}$ and $Y_{t}^{*}$ are all continuous for every $t>0$ and (50) holds, we can rewrite (45) as

$$
\begin{aligned}
& V\left(t, X_{t \wedge \tau}^{*}, Y_{t \wedge \tau}^{*}\right)=V(0, x, 0) \\
& +\int_{0}^{t \wedge \tau} e^{-\gamma Y_{t}^{*}}\left(w_{t}\left(t, X_{t}^{*}\right)+\mu w_{x}\left(t, X_{t}^{*}\right)+\frac{\sigma^{2}}{2} w_{x x}\left(t, X_{t}^{*}\right)\right) 1_{\left\{X_{t}^{*} \leq b(t)\right\}} d t \\
& +\sigma \int_{0}^{t \wedge \tau} e^{-\gamma Y_{t}^{*}} w_{x}\left(t, X_{t}^{*}\right) d W_{t} \\
& +\int_{0}^{t \wedge \tau} e^{-\gamma Y_{t}^{*}}\left(e^{-\beta t}\left(1-\gamma w\left(t, X_{t}^{*}\right)\right)-w_{x}\left(t, X_{t}^{*}\right)\right) 1_{\left\{X_{t}^{*}=b(t)\right\}} d\left(C^{*}\right)_{t}^{c} \\
& +V\left(0, x-(x-b(0))_{+},(x-b(0))_{+}\right)-V(0, x, 0) .
\end{aligned}
$$

The jump term in (51) obviously equals 0 for $x \leq b(0)$. In the case $x>b(0)$ we have by (47) that the last two terms in (51) equal

$$
\begin{aligned}
& V(0, b(0), x-b(0))-V(0, x, 0) \\
= & e^{-\gamma \vartheta}\left(1-\gamma w(0, \theta)-w_{x}(0, \theta)\right)(x-b(0))
\end{aligned}
$$

for some $\theta \in[b(0), x]$ and $\vartheta \in[0, x-b(0)]$. Since by proposition 2 we now have $1-\gamma w(0, \theta)-$ $w_{x}(0, \theta)=0$, the jump term again equals 0 . Taking expectation in (51) and using proposition 2 we obtain

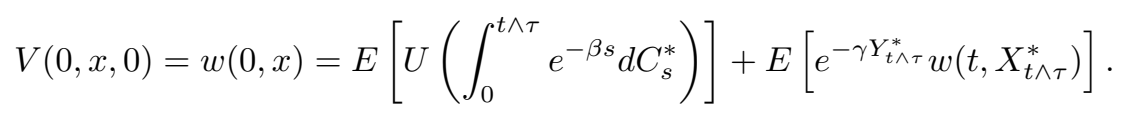

We note that

$$
e^{-\gamma Y_{t \wedge \tau}^{*}} w\left(t, X_{t \wedge \tau}^{*}\right) \rightarrow e^{-\gamma Y_{\tau}^{*}} w\left(t, X_{\tau}^{*}\right)=e^{-\gamma Y_{\tau}^{*}} w(t, 0)=0
$$

when $t \rightarrow+\infty$. Since $w$ is bounded by theorem 2 , the result follows by letting $t \rightarrow+\infty$ in (53) and using dominated convergence theorem.

\subsection{A series expansion}

\subsubsection{Ansatz and results}

In this section we try to find a more explicit description of the functions $d, b, w^{I}$, and $w^{I I}$, assuming they exist. Let us introduce the quantities

$$
r_{k}=\frac{-\mu+\sqrt{\mu^{2}+2 \beta \sigma^{2} k}}{\sigma^{2}}, \quad s_{k}=\frac{-\mu-\sqrt{\mu^{2}+2 \beta \sigma^{2} k}}{\sigma^{2}}, \quad(k \geq 1),
$$

and the functions

$$
g_{k}(x)=e^{r_{k} x}-e^{s_{k} x}, \quad(k \geq 1) .
$$


Then our ansatz is (c.f. also the discussion in Gerber and Shiu (2004), section 10.)

$$
b(t)=\sum_{n \geq 0} b_{n} e^{-n \beta t}, \quad d(t)=\sum_{n \geq 0} d_{n} e^{-n \beta t},
$$

and

$$
w^{I}(t, x)=\sum_{k \geq 1} c_{k} g_{k}(x) e^{-\beta t k}, \quad w^{I I}(t, x)=U\left(e^{-\beta t}(d(t)+x)\right) .
$$

Ignoring technicalities for the moment, we see that $w^{I}$ satisfies the PDE and the boundary condition, and $w^{I I}$ satisfies the ODE. We will see below that the smooth fit conditions translate into recursions for the sequences $\left(d_{n}\right)_{n \geq 0},\left(b_{n}\right)_{n \geq 0}$, and $\left(c_{n}\right)_{n \geq 1}$, that can be used to determine their values uniquely.

We use this ansatz numerically to get some ideas about the behaviour of $b$ and $w$, albeit the resulting conclusions are at present heuristic in nature, as we have been unable to prove, that the series converge and define sufficiently regular functions. Yet the calculations are rigorous in the domain of formal power series. Let us introduce the auxiliary functions

$$
p_{k}(x)=e^{r_{k} x}, \quad q_{k}(x)=e^{s_{k} x} \quad(k \geq 1),
$$

and

$$
\ell(t)=e^{-\beta t}(d(t)+b(t)), \quad u(t)=e^{-\gamma \ell(t)} .
$$

Then we have

$$
p_{k}(b(t))=\sum_{n \geq 0} p_{k n} e^{-\beta t n}, \quad q_{k}(b(t))=\sum_{n \geq 0} q_{k n} e^{-\beta t n},
$$

and

$$
\ell(t)=\sum_{n \geq 0} \ell_{n} e^{-\beta t n}, \quad u(t)=\sum_{n \geq 0} u_{n} e^{-\beta t n} .
$$

We will also use

$$
g_{k}^{(j)}(b(t))=\sum_{n \geq 0} g_{j k n} e^{-\beta t n} \quad(j \geq 0, k \geq 1),
$$

where $g_{k}^{(j)}$ denotes the $j$-th derivative of the function $g_{k}$. We first show, that the auxiliary quantities satisfy

$$
\begin{aligned}
& p_{k 0}=e^{r_{k} b_{0}}, \quad p_{k n}=\frac{r_{k}}{n} \sum_{j=1}^{n} j b_{j} p_{k, n-j} \quad(k \geq 1, n \geq 1) \\
& q_{k 0}=e^{s_{k} b_{0}}, \quad q_{k n}=\frac{s_{k}}{n} \sum_{j=1}^{n} j b_{j} q_{k, n-j} \quad(k \geq 1, n \geq 1) \\
& g_{j k n}=r_{k}^{j} p_{k n}-s_{k}^{j} q_{k n} \quad(j \geq 0, k \geq 1, n \geq 0) \\
& \ell_{n}=d_{n-1}+b_{n-1} \quad(n \geq 1) \\
& u_{0}=1, \quad u_{n}=-\frac{\gamma}{n} \sum_{j=1}^{n} j \ell_{j} u_{n-j} \quad(n \geq 1) .
\end{aligned}
$$

For the purposes of this section it will be convenient to consider the equations

$$
w^{I}(t, b(t))=w^{I I}(t, b(t)), \quad w_{x}^{I}(t, b(t))=w_{x}^{I I}(t, b(t)), \quad w_{x x}^{I}(t, b(t))=w_{x x}^{I I}(t, b(t)),
$$


which are equivalent to the smooth fit conditions. Comparing coefficients this system leads to

$$
\begin{aligned}
& c_{1} g_{010}=-\frac{u_{1}}{\gamma}, \quad c_{1} g_{110}=1, \quad c_{1} g_{210}=0 \\
& \sum_{k=1}^{n} c_{k} g_{0, k, n-k}=-\frac{u_{n}}{\gamma}, \quad \sum_{k=1}^{n} c_{k} g_{1, k, n-k}=u_{n-1}, \quad \sum_{k=1}^{n} c_{k} g_{2, k, n-k}=-\gamma u_{n-2} \quad(n \geq 2) .
\end{aligned}
$$

This system admits a unique solution that can be computed step by step from

$$
\begin{aligned}
& b_{0}=\frac{1}{r_{1}-s_{1}} \ln \left(\frac{s_{1}^{2}}{r_{1}^{2}}\right), \quad c_{1}=\frac{1}{g_{110}}, \quad d_{0}=\frac{\mu}{\beta}-b_{0}, \quad d_{1}=-\frac{\sigma^{2} \gamma}{4 \beta}-\frac{b_{1}}{2}, \\
& c_{n}=\frac{1}{g_{1 n 0}}\left[u_{n-1}-\frac{c_{1}}{n-1} \sum_{j=1}^{n-2} j b_{j} g_{2,1, n-1-j}-\sum_{k=2}^{n-1} c_{k} g_{1, k, n-k}\right] \quad(n \geq 2) \\
& b_{n}=-\frac{1}{c_{1} g_{310}}\left[\gamma u_{n-1}+\frac{c_{1}}{n} \sum_{j=1}^{n-1} j b_{j} g_{3,1, n-j}+\sum_{k=2}^{n+1} c_{k} g_{2, k, n+1-k}\right] \quad(n \geq 1) \\
& d_{n}=-\frac{b_{n}}{n+1} \quad(n \geq 2)
\end{aligned}
$$

In this system the correct order of evaluation is not specified. It is as follows: Set $u_{0}=1$, compute $b_{0}$ and $d_{0}$. From $b_{0}$ we can calculate $p_{10}, q_{10}$ and $g_{j 10}$ for $j=0, \ldots, 3$. Next we compute $c_{1}$. Then we perform for each $n \geq 2$ the calculations for

1. $p_{n 0}, q_{n 0}$, and $g_{j n 0}$ for $j=0, \ldots, 3$,

2. $\ell_{n-1}$ and $u_{n-1}$,

3. for each $k=2, \ldots, n-1$ compute $p_{k, n-k}, q_{k, n-k}$, and $g_{j, k, n-k}$ for $j=0, \ldots, 3$,

4. $c_{n}, b_{n-1}$, and $d_{n-1}$,

5. $p_{1, n-1}, q_{1, n-1}$, and $g_{j, 1, n-1}$ for $j=0, \ldots, 3$.

\subsubsection{Detailed calculations}

Let us first derive series for $w^{I}(t, b(t)), w_{x}^{I}(t, b(t))$, and $w_{x x}^{I}(t, b(t))$. From

$$
p_{k}\left(b_{0}\right)=e^{r_{k} b_{0}}, \quad p_{k}(b(t))^{\prime}=b^{\prime}(t) p_{k}^{\prime}(b(t))
$$

and

$$
q_{k}\left(b_{0}\right)=e^{s_{k} b_{0}}, \quad q_{k}(b(t))^{\prime}=b^{\prime}(t) q_{k}^{\prime}(b(t))
$$

we obtain

$$
p_{k 0}=e^{r_{k} b_{0}}, \quad p_{k n}=\frac{r_{k}}{n} \sum_{j=1}^{n} j b_{j} p_{k, n-j} \quad(k \geq 1, n \geq 1)
$$

and

$$
q_{k 0}=e^{s_{k} b_{0}}, \quad q_{k n}=\frac{s_{k}}{n} \sum_{j=1}^{n} j b_{j} q_{k, n-j} \quad(k \geq 1, n \geq 1) .
$$


Let us write for simplicity $g_{k n}, g_{k n}^{\prime}, g_{k n}^{\prime \prime}, g_{k n}^{\prime \prime \prime}$ instead of $g_{0 k n}, g_{1 k n}, g_{2 k n}, g_{3 k n}$. With this we have

$$
\begin{aligned}
g_{k}(b(t)) & =\sum_{n \geq 0} g_{k n} e^{-\beta t n}, & g_{k}^{\prime}(b(t)) & =\sum_{n \geq 0} g_{k n}^{\prime} e^{-\beta t n}, \\
g_{k}^{\prime \prime}(b(t)) & =\sum_{n \geq 0} g_{k n}^{\prime \prime} e^{-\beta t n}, & g_{k}^{\prime \prime \prime}(b(t)) & =\sum_{n \geq 0} g_{k n}^{\prime \prime \prime} e^{-\beta t n},
\end{aligned}
$$

where

$$
g_{k n}=p_{k n}-q_{k n}, \quad g_{k n}^{\prime}=r_{k} p_{k n}-s_{k} q_{k n}, \quad g_{k n}^{\prime \prime}=r_{k}^{2} p_{k n}-s_{k}^{2} q_{k n}, \quad g_{k n}^{\prime \prime \prime}=r_{k}^{3} p_{k n}-s_{k}^{3} q_{k n} .
$$

This implies then

$$
\begin{aligned}
& w^{I}(t, b(t))=\sum_{n \geq 1}\left(\sum_{k=1}^{n} c_{k} g_{k, n-k}\right) e^{-\beta t n}, \\
& w_{x}^{I}(t, b(t))=\sum_{n \geq 1}\left(\sum_{k=1}^{n} c_{k} g_{k, n-k}^{\prime}\right) e^{-\beta t n}, \\
& w_{x x}^{I}(t, b(t))=\sum_{n \geq 1}\left(\sum_{k=1}^{n} c_{k} g_{k, n-k}^{\prime \prime}\right) e^{-\beta t n} .
\end{aligned}
$$

Next let us derive series for $w^{I I}(t, b(t)), w_{x}^{I I}(t, b(t))$, and $w_{x x}^{I I}(t, b(t))$. Let us introduce the auxiliary function

$$
\ell(t)=e^{-\beta t}(d(t)+b(t)) .
$$

Clearly

$$
\ell(t)=\sum_{n \geq 1} \ell_{n} e^{-\beta t n}
$$

where

$$
\ell_{n}=d_{n-1}+b_{n-1}, \quad(n \geq 1) .
$$

Let us also introduce the function $u(x)=U^{\prime}(x)$, i.e.,

$$
u(x)=e^{-\gamma x} .
$$

Then we have

$$
u(\ell(t))=\sum_{n \geq 0} u_{n} e^{-\beta t n}
$$

From

$$
u(0)=1, \quad u(\ell(t))^{\prime}=\ell^{\prime}(t) u^{\prime}(\ell(t)),
$$

and observing $u^{\prime}(x)=-\gamma u(x)$, we obtain

$$
u_{0}=1, \quad u_{n}=-\frac{\gamma}{n} \sum_{j=1}^{n} j \ell_{j} u_{n-j} \quad(n \geq 1) .
$$

From

$$
w^{I I}(t, b(t))=\frac{1-u(\ell(t))}{\gamma}, \quad w_{x}^{I I}(t, b(t))=e^{-\beta t} u(\ell(t)), \quad w_{x x}^{I I}(t, b(t))=-\gamma e^{-2 \beta t} u(\ell(t)),
$$


we obtain

$$
\begin{aligned}
& w^{I I}(t, b(t))=-\frac{1}{\gamma} \sum_{n \geq 1} u_{n} e^{-\beta t n}, \\
& w_{x}^{I I}(t, b(t))=\sum_{n \geq 1} u_{n-1} e^{-\beta t n}, \\
& w_{x x}^{I I}(t, b(t))=-\gamma \sum_{n \geq 2} u_{n-2} e^{-\beta t n} .
\end{aligned}
$$

Comparing coefficients yields a system of recursions. For $n=1$ we have to solve

$$
c_{1} g_{10}=-\frac{u_{1}}{\gamma}, \quad c_{1} g_{10}^{\prime}=1, \quad c_{1} g_{10}^{\prime \prime}=0
$$

and for $n \geq 2$

$$
\sum_{k=1}^{n} c_{k} g_{k, n-k}=-\frac{u_{n}}{\gamma}, \quad \sum_{k=1}^{n} c_{k} g_{k, n-k}^{\prime}=u_{n-1}, \quad \sum_{k=1}^{n} c_{k} g_{k, n-k}^{\prime \prime}=-\gamma u_{n-2} .
$$

Let us first see, how $\left(d_{0}, b_{0}, c_{1}\right)$ can be determined from (88). Obviously we must have $g_{10}^{\prime \prime}=0$, which gives

$$
b_{0}=\frac{1}{r_{1}-s_{1}} \ln \left(\frac{s_{1}^{2}}{r_{1}^{2}}\right) .
$$

Knowing the value $b_{0}$ we can compute $g_{10}$ and $g_{10}^{\prime}$. Next we get

$$
c_{1}=\frac{1}{g_{10}^{\prime}} .
$$

Using $u_{1}=-\gamma\left(d_{0}+b_{0}\right)$ we obtain

$$
d_{0}=c_{1} g_{10}-b_{0} .
$$

Next let us consider $n \geq 2$, and see, how $\left(d_{n-1}, b_{n-1}, c_{n}\right)$ can be determined from (89), assuming $\left(d_{k-1}, b_{k-1}, c_{k}\right)$ and all associated auxiliary quantities have been computed for $k=1, \ldots, n-1$. To that purpose we consider (69) and (70) for the computation of $p_{1, n-1}$ resp. $q_{1, n-1}$ and extract the last summand, i.e., we can write

$$
p_{1, n-1}=b_{n-1} r_{1} p_{10}+\frac{1}{n-1} \sum_{j=1}^{n-2} j b_{j} r_{1} p_{1, n-1-j}
$$

and

$$
q_{1, n-1}=b_{n-1} s_{1} q_{10}+\frac{1}{n-1} \sum_{j=1}^{n-2} j b_{j} s_{1} q_{1, n-1-j} .
$$

Using those expressions, and observing $g_{10}^{\prime \prime}=0$ from above, we obtain

$$
g_{1, n-1}^{\prime}=\frac{1}{n-1} \sum_{j=1}^{n-2} j b_{j} g_{1, n-1-j}^{\prime \prime}
$$

Similarly

$$
g_{1, n-1}^{\prime \prime}=b_{n-1} g_{10}^{\prime \prime \prime}+\frac{1}{n-1} \sum_{j=1}^{n-2} j b_{j} g_{1, n-1-j}^{\prime \prime \prime}
$$


Let us extract the first and the last term from the sum in the second equation of (89),

$$
c_{1} g_{1, n-1}^{\prime}+c_{n} g_{n 0}^{\prime}+\sum_{k=2}^{n-1} c_{k} g_{k, n-k}^{\prime}=u_{n-1} .
$$

Plugging (95) into this equation yields

$$
c_{n}=\frac{1}{g_{n 0}^{\prime}}\left[u_{n-1}-\frac{c_{1}}{n-1} \sum_{j=1}^{n-2} j b_{j} g_{1, n-1-j}^{\prime \prime}-\sum_{k=2}^{n-1} c_{k} g_{k, n-k}^{\prime}\right] .
$$

Here we use that $\beta>0$ and $\sigma>0$ implies $r_{n}>0$ and $s_{n}<0$, and thus $g_{n 0}^{\prime}=r_{n} e^{r_{n} b_{0}}-s_{n} e^{s_{n} b_{0}}>0$. Now let us extract the first term from the sum in the third equation of (89),

$$
c_{1} g_{1, n-1}^{\prime \prime}+\sum_{k=2}^{n} c_{k} g_{k, n-k}^{\prime \prime}=-\gamma u_{n-2} .
$$

Plugging (96) into this equation yields

$$
b_{n-1}=-\frac{1}{c_{1} g_{10}^{\prime \prime \prime}}\left[\gamma u_{n-2}+\frac{c_{1}}{n-1} \sum_{j=1}^{n-2} j b_{j} g_{1, n-1-j}^{\prime \prime \prime}+\sum_{k=2}^{n} c_{k} g_{k, n-k}^{\prime \prime}\right] .
$$

Finally let us extract the last term in the sum (83),

$$
u_{n}=-\gamma\left[\frac{1}{n} \sum_{j=1}^{n-1} j \ell_{j} u_{n-j}+\ell_{n}\right] .
$$

Plugging this expression into the first equation of (89) gives

$$
d_{n-1}=-b_{n-1}-\frac{1}{n} \sum_{j=1}^{n-1} j \ell_{j} u_{n-j}+\sum_{k=1}^{n} c_{k} g_{k, n-k} .
$$

It turns out, that this expression can be simplified considerably, namely we have

$$
d_{0}=\frac{\mu}{\beta}-b_{0}, \quad d_{1}=-\frac{\sigma^{2} \gamma}{4 \beta}-\frac{b_{1}}{2}, \quad d_{n}=-\frac{b_{n}}{n+1} \quad(n \geq 2) .
$$

This result is quite intricate to prove from the recursions (89), but follows immediately from plugging the series ansatz into the first order differential equation for $d$ in terms of $b$, that has been derived above.

In Figure 2 we give Mathematica code to compute the coefficients: The code uses the ability of the Mathematica system to figure out, which recursion is to be evaluated when. The correct order of evaluation is specified explicitly in the code in Figure 3.

\subsubsection{Some numerical evidence}

In addition to the series ansatz we experimented with an Euler-type scheme for an integrodifferential equation, which one gets by differentiating our basic integral equation (25) w.r.t. $t$.

$$
b^{\prime}(t)=\frac{I_{4}(t)+I_{6}(t)-I_{1}(t)-I_{3}(t)}{I_{2}(t)-I_{5}(t)},
$$




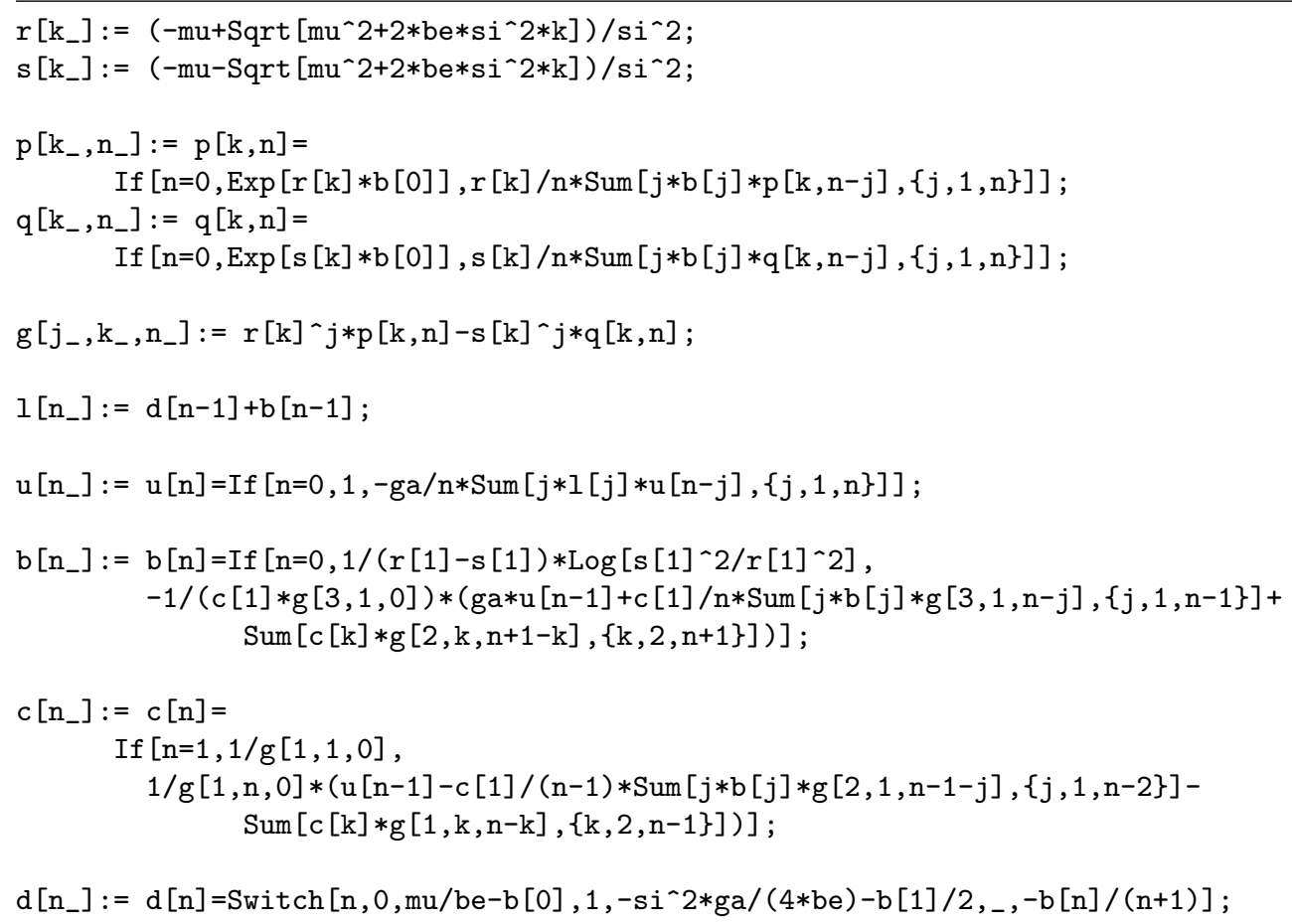

Figure 2: Mathematica code for computing the coefficients with implicit evaluation order.

where

$$
\begin{aligned}
& I_{1}(t)=\frac{\sigma^{2}}{2} \int_{0}^{\infty} G_{x}(r, b(r+t), b(t)) b^{\prime}(r+t) \tilde{u}(r+t) d r \\
& I_{2}(t)=\frac{\sigma^{2}}{2} \int_{0}^{\infty} G_{x^{\prime}}(r, b(r+t), b(t)) \tilde{u}(r+t) d r \\
& I_{3}(t)=\frac{\sigma^{2}}{2} \int_{0}^{\infty} G(r, b(r+t), b(t)) \tilde{u}^{\prime}(r+t) d r \\
& I_{4}(t)=-\int_{0}^{\infty} G(r, b(r+t), b(t)) b^{\prime}(r+t) \rho^{\prime}(r+t) b(r+t) d r \\
& I_{5}(t)=-\int_{0}^{\infty} \int_{0}^{b(r+t)} x G_{x^{\prime}}(r, x, b(t)) \rho^{\prime}(r+t) d x d r \\
& \left.I_{6}(t)=\frac{\partial}{\partial t} \int_{0}^{A(r)} x G(r, x, B) \rho^{\prime}(r+t) d x d r\right)\left.\right|_{(A(r)=b(r+t), B=b(t))} .
\end{aligned}
$$

We start from the approximation $b(T) \approx b_{0}$ for some large $T$ and proceed backwards in time towards $t=0$. We do not provide any mathematical analysis of this approach, so it remains experimental, but the following graph (Figure 4) illustrates a fairly good agreement of both methods. 


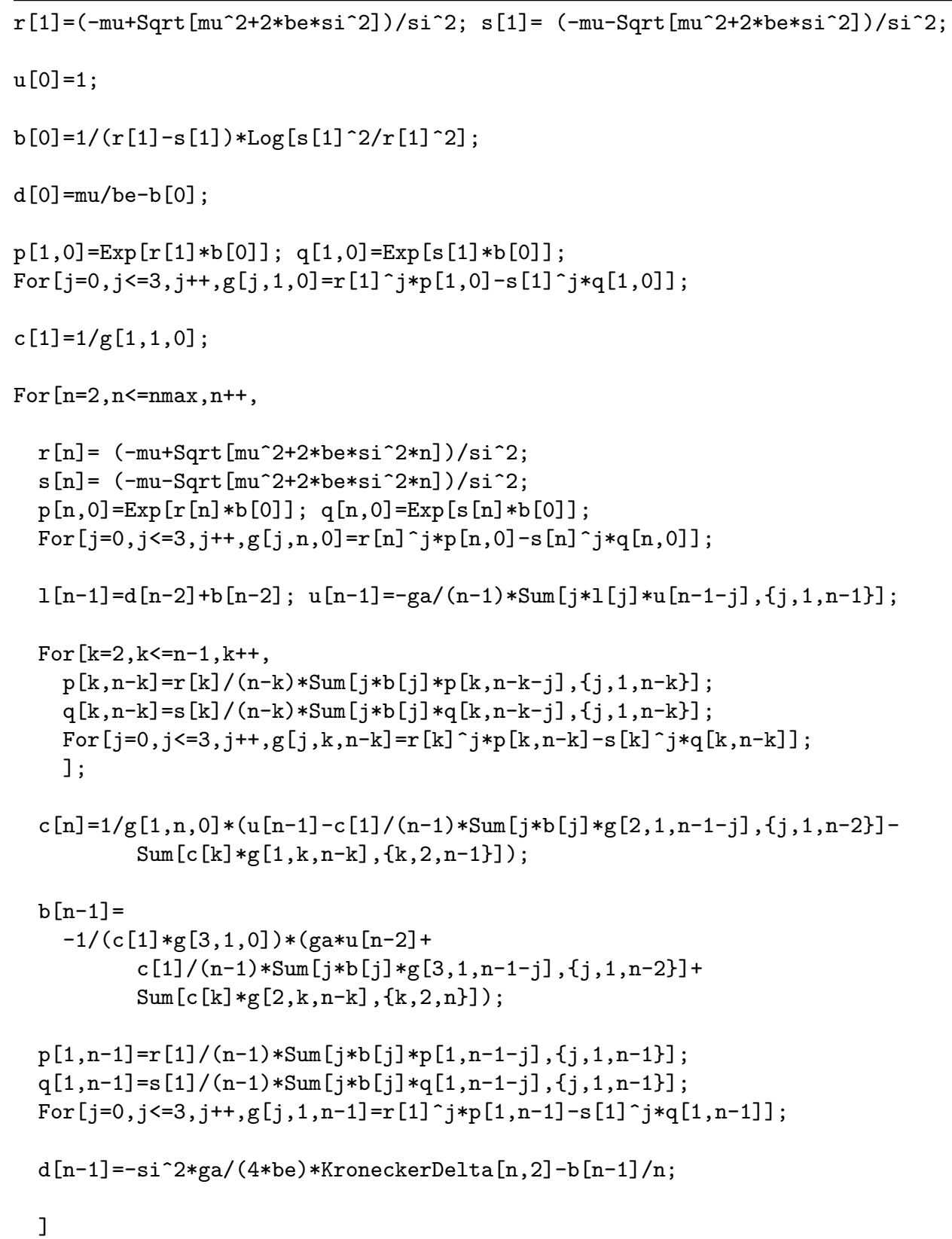

Figure 3: Mathematica code to evaluate the coefficients with explicit order of evaluations. 


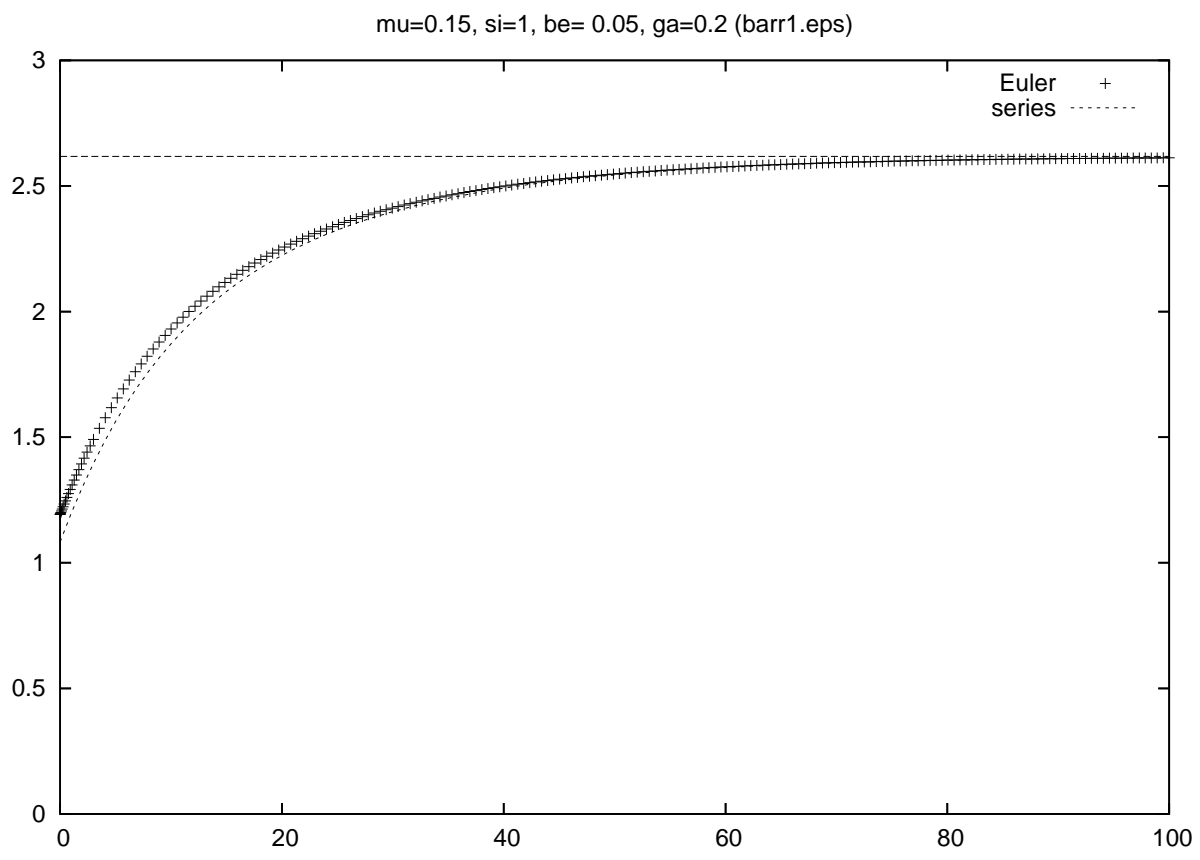

Figure 4: A comparison of the approximations of the barrier $b(t)$ obtained by the series ansatz and a backward Euler scheme

\section{Proof of proposition 1}

Taking $N$ large, to be chosen later, we first perform the transformations

$$
\begin{aligned}
\tau & =e^{-\frac{t}{N}}, \\
z & =\frac{x}{b(\tau)}, \\
u(\tau, z) & =w(\tau, z)-z D(\tau) .
\end{aligned}
$$

This gives

$$
\begin{aligned}
\tau u_{\tau} & =\frac{N \sigma^{2}}{2 b^{2}(\tau)} u_{z z}+\frac{\mu N+z \tau b^{\prime}(\tau)}{b(\tau)} u_{z}+f(\tau, z), \\
u(0, z) & =u(\tau, 0)=u(\tau, 1)=0,
\end{aligned}
$$

where

$$
f(\tau, z)=\frac{\mu N+z \tau b^{\prime}(\tau)}{b(\tau)} D(\tau)-z \tau D^{\prime}(\tau) .
$$

This equation has two difficulties:

- the function $\tau$ in front of $u_{\tau}$,

- the fact that we do not know that the function in front of $u_{z}$ is Hölder continuous, which is assumed in most of the literature. 
So we have to use a somewhat cumbersome method, where we apply two different results from earlier papers to get an existence and regularity proof. We will first use the paper Friedman and Schuss (1971) to analyze our equation (106). Equation (106) can be written in the form of Friedman and Schuss (1971), namely

$$
c_{1}(\tau) \frac{d u}{d \tau}+c_{2}(\tau) A(\tau) u=f(\tau) \quad \text { in }(0,1]
$$

in some Hilbert space $X$. We set the Hilbert space $X$ of Friedman and Schuss (1971) to be $L^{2}(0,1)$ and $D_{A}=H^{2} \cap H_{0}^{1}$, where $H^{k}$ and $H_{0}^{k}$ always mean Sobolev spaces (see e.g. Renardy and Rogers (1993)). We use theorems 1.1, 2.1 and 3.1 of Friedman and Schuss (1971) in order to get a solution

$$
u(\tau, z) \in C\left((0,1], H^{2} \cap H_{0}^{1}\right) .
$$

The following remarks are in order:

The domain of the operator $A$ is obviously independent of $\tau$, and the fact that $A$ generates an analytic semigroup (see Renardy and Rogers (1993)) guaranties the validity of conditions $(i)$ and $(i i)$ of definition 1.1. We also have $\tau \in \mathcal{C}_{0}$ of Friedman and Schuss (1971). Finally we check that $A(\tau)$ satisfies the condition $\left(A C_{0}\right)$ of definition 1.3. We will prove

$$
\int_{0}^{1} u\left(-\frac{N \sigma^{2}}{2 b^{2}(\tau)} u_{z z}-\frac{\mu N+z \tau b^{\prime}(\tau)}{b(\tau)} u_{z}\right) d z \geq \gamma \int_{0}^{1}|u|^{2} d z,
$$

for all $u \in C_{0}^{\infty}[0,1]$ and some $\gamma>1$. We start by partially integrating 1.h.s.

$$
\begin{aligned}
& \int_{0}^{1} u\left(-\frac{N \sigma^{2}}{2 b^{2}(\tau)} u_{z z}-\frac{\mu N+z \tau b^{\prime}(\tau)}{b(\tau)} u_{z}\right) d z \\
= & \frac{N \sigma^{2}}{2 b^{2}(\tau)} \int_{0}^{1} u_{z}^{2} d z-\frac{\mu N}{b(\tau)} \int_{0}^{1} u u_{z} d z-\frac{\tau b^{\prime}(\tau)}{b(\tau)} \int_{0}^{1} z u u_{z} d z \\
= & \frac{N \sigma^{2}}{2 b^{2}(\tau)} \int_{0}^{1} u_{z}^{2} d z-\frac{\tau b^{\prime}(\tau)}{b(\tau)} \int_{0}^{1} z u u_{z} d z .
\end{aligned}
$$

It is easy to verify via partial integration

$$
\int_{0}^{1} z u u_{z} d z=-\frac{1}{2} \int_{0}^{1} u^{2} d z
$$

so the l.h.s. of (110) equals

$$
\frac{N \sigma^{2}}{2 b^{2}(\tau)} \int_{0}^{1} u_{z}^{2} d z+\frac{\tau b^{\prime}(\tau)}{2 b(\tau)} \int_{0}^{1} u^{2} d z .
$$

We next use Poincaré's inequality to note that for some constant $C>1$

$$
\int_{0}^{1}\left(u^{2}+u_{z}^{2}\right) d z \leq C \int_{0}^{1} u_{z}^{2} d z
$$

thus implying

$$
\int_{0}^{1} u^{2} d z \leq(C-1) \int_{0}^{1} u_{z}^{2} d z
$$


This means that there exists $\alpha>0$ such that

$$
\int_{0}^{1} u_{z}^{2} d z \geq \alpha \int_{0}^{1} u^{2} d z
$$

so we can estimate the l.h.s of (110) by

$$
\int_{0}^{1} u\left(-\frac{N \sigma^{2}}{2 b^{2}(\tau)} u_{z z}-\frac{\mu N+z \tau b^{\prime}(\tau)}{b(\tau)} u_{z}\right) d z \geq\left(\frac{\alpha N \sigma^{2}}{2 b^{2}(\tau)}+\frac{\tau b^{\prime}(\tau)}{2 b(\tau)}\right) \int_{0}^{1} u^{2} d z .
$$

We can now easily choose $N$ large enough to obtain

$$
\frac{\alpha N \sigma^{2}}{2 b^{2}(\tau)}+\frac{\tau b^{\prime}(\tau)}{2 b(\tau)}>1 \quad \forall \tau \in[0,1]
$$

The only problem is that neither $A(\tau)$ nor $f(\tau, z)$ is Hölder continuous, where the former is used in theorems 1.1, 2.1 and 3.1, and the latter in theorem 1.1. But checking the proofs carefully, one sees that these Hölder continuities are only used when they assert the existence of some approximating classical solution. (The approximating equations do not have a zero in the function in front of the time derivative, so standard literature is used, namely reference [4] in Friedman and Schuss (1971).) Since we do not have Hölder continuity of $A$ and $f$ as in reference [4] in Friedman and Schuss (1971), we use the paper Buttu (1992), where a classical solution without these assumptions is shown (theorem 2.2). In order to use Buttu (1992) safely, one has to show that our operator generates an analytic semigroup on the continuous interpolation spaces $D_{A}(\vartheta, \infty)$ introduced in Da Prato and Grisvard (1979), which she uses in her approach. But this is shown in Amann (1993), Section 6. All this gives us equation (109).

Finally, we note that in all Banach spaces $Y$, such that $H^{s_{1}}, s_{1}<\frac{1}{2}$ is densely and continuously injected in $Y$ and $Y \subset H^{s_{2}}, s_{2}<\frac{1}{2}$ (e.g. the relevant interpolation space of Buttu (1992) can be taken for $Y$ ) we have

$$
\|f(\tau, \cdot)\|_{Y}=\mathcal{O}\left(\tau^{N^{\prime}}\right)
$$

where $N^{\prime}$ becomes large if $N$ becomes large. The reason is that one has $H_{0}^{s}=H^{s}$ for $s<\frac{1}{2}$ (see Lions and Magenes (1972), Theorem 11.1), so our $f$ fulfills $f(\tau) \in H_{0}^{s}$ for $s<\frac{1}{2}$ and lies therefore also in the proper interpolation spaces of Buttu (1992). Equation (111) easily follows from the structure of our $f$.

Applying the estimates in Buttu (1992), Theorem 2.2 one easily gets

$$
\|u(\tau)\|_{H^{2} \cap H_{0}^{1}}=\mathcal{O}\left(\tau^{N^{\prime}}\right),
$$

which together with (109) yields

$$
u(\tau, z) \in C\left([0,1], H^{2} \cap H_{0}^{1}\right) .
$$

The assertion $w_{x} \in C(M)$ in the theorem is now an easy consequence of Sobolev imbedding theorem and re-transformation to the variables $w, t, x$.

Once we have proved this, the assertions concerning $w_{t}$ and $w_{x x}$ are easy to see.

The fact that $w_{t}$ exist and is continuous in the interior follows from the fact that $w$ solves a diffusion equation with constant coefficients (and is therefore $C^{\infty}$ ). The existence and continuity of $w_{t}$ at the boundary is a consequence of the fact that $w_{x}$ is continuous up to the boundary, and $b(t)$ and $w(t, b(t))=D(t)$ are $C^{1}([0, \infty))$. Now $w_{x x}$ exist and is continuous up to the boundary, because of the validity of our PDE in the interior and the already proved assertion for $w_{t}$ and $w_{x}$. 


\section{Proof of Proposition 2}

Denote by $I=\left\{(t, x) \in \mathbb{R}_{+}^{2} ; t \geq 0, x \leq b(t)\right\}$ and $I I=\left\{(t, x) \in \mathbb{R}_{+}^{2} ; t \geq 0, x>b(t)\right\}$. As it is already noted in section 1.2 the general solution of (ii) is given by $w^{I I}(t, x)=U\left(e^{-\beta t}(x+d(t))\right)$. We show that by choosing

$$
d(t)=e^{\beta t} \int_{t}^{\infty}\left(\mu e^{-\beta s}-\frac{\gamma \sigma^{2}}{2} e^{-2 \beta s}-\beta e^{-\beta s} b(s)\right) d s .
$$

(vii) is satisfied: Plugging $U\left(e^{-\beta t}(x+d(t))\right)$ into (vii) gives

$$
e^{-\gamma e^{-\beta t}(b(t)+d(t))}\left(-\beta e^{-\beta t}(b(t)+d(t))+e^{-\beta t} d^{\prime}(t)+\mu e^{-\beta t}-\frac{\gamma \sigma^{2}}{2} e^{-2 \beta t}\right)=0,
$$

and after simplification

$$
d^{\prime}(t)=-\mu+\frac{\gamma \sigma^{2}}{2} e^{-\beta t}+\beta(b(t)+d(t)) .
$$

It is easy to see that $d(t)$ given by (114) is a particular solution of this ODE. (Choosing a different particular solution would yield a $d(t) \sim$ const. $e^{\beta t}$, hence $w(t, b(t)) \nrightarrow 0$ for $t \rightarrow \infty$.)

Set now $D(t) \equiv w^{I I}(t, b(t))=U\left(e^{-\beta t}(b(t)+d(t))\right)$. This gives

$$
\begin{array}{r}
D^{\prime}(t)=U^{\prime}\left(e^{-\beta t}(b(t)+d(t))\right) e^{-\beta t}\left(b^{\prime}(t)+d^{\prime}(t)-\beta(b(t)+d(t))\right) \\
=U^{\prime}\left(e^{-\beta t}(b(t)+d(t))\right) e^{-\beta t}\left(b^{\prime}(t)-\mu+\frac{\gamma \sigma^{2}}{2} e^{-\beta t}\right) .
\end{array}
$$

Hence

$$
D^{\prime}(t) \sim-\mu e^{-\beta t} .
$$

We solve now the boundary problem 31 in Proposition 1 to get $w^{I}$. Therefore (i), (iii), (iv) and (v) of our assertion is now true. The last smooth fitting condition we have to satisfy is (vi). The idea is the following: First find a formula for $w_{x}^{I}$ at the boundary $(t, b(t))$, depending on the boundary data of our boundary value problem. This is done using Green's function (actually we will transform to a different dependent variable called $u$.) Finally we will show that $w_{x}^{I I}$ (or rather $u_{x}^{I I}$ ) satisfies the formula mentioned above, if our basic integral equation (25) has a solution.

From (114) we see that $d(t)$ is determined by the values of $b(s)$ for $s \geq t$. We denote such dependence by $d(t)=d[b(s) ; s \geq t]$. It follows that $D(t)=D[b(s) ; s \geq t]$. By assumption $(i)$ we also have $D(t)=w(t, b(t))$. Let

$$
v(t, x):=w(t, x) e^{\frac{\mu}{\sigma^{2}} x-\frac{\mu^{2}}{2 \sigma^{2}} t} .
$$

Using the fact that $w$ solves the boundary problem of proposition 1, elementary calculation shows that

in region $I$. Define

$$
v_{t}+\frac{\sigma^{2}}{2} v_{x x}=0
$$

$$
M(t):=v(t, b(t))=D(t) e^{\frac{\mu}{\sigma^{2}} b(t)-\frac{\mu^{2}}{2 \sigma^{2}} t},
$$

and note that $M(t)=M[b(s) ; s \geq t]$. Furthermore, let

$$
\begin{aligned}
\rho(t) & =\frac{M(t)}{b(t)}, \\
u(t, x) & =v(t, x)-x \rho(t), \\
g(t, x) & =-x \rho^{\prime}(t) .
\end{aligned}
$$


We see that $\rho(t)=\rho[b(s) ; s \geq t]$. We also note that $M(t)$ and $\rho(t)$ are in $C^{1}$ and

$$
-M^{\prime}(t) \sim \text { const. } e^{-\left(\beta+\frac{\mu^{2}}{2 \sigma^{2}}\right) t}, \quad-\rho^{\prime}(t) \sim \text { const. } e^{-\left(\beta+\frac{\mu^{2}}{2 \sigma^{2}}\right) t}
$$

for some positive constants. Easy calculation shows that $u$ solves following boundary problem

$$
\begin{aligned}
L u & :=u_{t}+\frac{\sigma^{2}}{2} u_{x x}=g(t, x) \quad \text { in } I \\
u(t, 0) & =0 \\
u(t, b(t)) & =0 \\
u(\infty, x) & =0 .
\end{aligned}
$$

By proposition 1 we know that this problem has a solution. We try to find an integro differential equation for $b$ using a representation of this solution involving Green's function. Fix $t>t^{\prime}>0$. Let

and let $G$ be such that

$$
L^{*} u=-u_{t}+\frac{\sigma^{2}}{2} u_{x x}
$$

$$
L^{*} G\left(t-t^{\prime}, x, x^{\prime}\right)=-\delta\left(x-x^{\prime}\right) \delta\left(t-t^{\prime}\right), \quad x \geq 0, x^{\prime}>0
$$

where $\delta$ is Dirac measure at 0 . We use the mirror method to find a Green's function, which fulfills the symmetry relation (129) below.

$$
G\left(t-t^{\prime}, x, x^{\prime}\right)=\frac{1}{\sigma \sqrt{2 \pi\left(t-t^{\prime}\right)}} \theta\left(t-t^{\prime}\right)\left(e^{-\frac{\left(x-x^{\prime}\right)^{2}}{2 \sigma^{2}\left(t-t^{\prime}\right)}}-e^{-\frac{\left(x+x^{\prime}\right)^{2}}{2 \sigma^{2}\left(t-t^{\prime}\right)}}\right),
$$

where $\theta$ is the Heaviside function. Note that

$$
G\left(t-t^{\prime}, 0, x^{\prime}\right)=0 .
$$

Next we consider an integral of the form

$$
\int_{t^{\prime}}^{\infty} \int_{0}^{b(t)}\left(G L u-u L^{*} G\right) d x d t
$$

On one hand, (130) is equal to

$$
\int_{t^{\prime}}^{\infty} \int_{0}^{b(t)} G\left(t-t^{\prime}, x, x^{\prime}\right) g(t, x) d x d t+u\left(t^{\prime}, x^{\prime}\right)
$$

Note that this relation holds also for $x^{\prime}=b\left(t^{\prime}\right)$, since there we have $u\left(t^{\prime}, b\left(t^{\prime}\right)\right)=0$. On the other hand, (130) equals

$$
\int_{t^{\prime}}^{\infty} \int_{0}^{b(t)} G\left(u_{t}+\frac{\sigma^{2}}{2} u_{x x}\right) d x d t-\int_{t^{\prime}}^{\infty} \int_{0}^{b(t)} u\left(-G_{t}+\frac{\sigma^{2}}{2} G_{x x}\right) d x d t
$$

By partial integration we have

$$
\begin{aligned}
& \int_{0}^{b(t)} \frac{\sigma^{2}}{2} G u_{x x} d x=\left.\frac{\sigma^{2}}{2} G u_{x}\right|_{0} ^{b(t)}-\int_{0}^{b(t)} \frac{\sigma^{2}}{2} G_{x} u_{x} d x, \\
& \int_{0}^{b(t)} \frac{\sigma^{2}}{2} u G_{x x} d x=\left.\frac{\sigma^{2}}{2} u G_{x}\right|_{0} ^{b(t)}-\int_{0}^{b(t)} \frac{\sigma^{2}}{2} G_{x} u_{x} d x .
\end{aligned}
$$


We also have

$$
\int_{t^{\prime}}^{\infty} \int_{0}^{b(t)}\left(G u_{t}+G_{t} u\right) d x d t=0
$$

In order to prove it we note that, since $b^{\prime}(t) G\left(t-t^{\prime}, b(t), x^{\prime}\right) u(t, b(t))=0$ by (125), the l.h.s. above equals

$$
\begin{aligned}
\int_{t^{\prime}}^{\infty} \int_{0}^{b(t)} \frac{\partial}{\partial t}(G u) d x d t & =\int_{t^{\prime}}^{\infty} \frac{\partial}{\partial t}\left(\int_{0}^{b(t)} G u d x\right) d t \\
& =\left.\int_{0}^{b(t)} G\left(t-t^{\prime}, x, x^{\prime}\right) u(t, x) d x\right|_{t=t^{\prime}} ^{\infty}=0
\end{aligned}
$$

where the last step follows from $u(\infty, x)=0$ and $\lim _{t \rightarrow t^{\prime}} G\left(t-t^{\prime}, x, x^{\prime}\right)=0$. Plugging (133) and (134) into (132), we have that (130) equals

$$
\begin{aligned}
& \int_{t^{\prime}}^{\infty}\left(\left.\frac{\sigma^{2}}{2} G u_{x}\right|_{0} ^{b(t)}-\int_{0}^{b(t)} \frac{\sigma^{2}}{2} G_{x} u_{x} d x\right) d t \\
& -\int_{t^{\prime}}^{\infty}\left(\left.\frac{\sigma^{2}}{2} u G_{x}\right|_{0} ^{b(t)}-\int_{0}^{b(t)} \frac{\sigma^{2}}{2} G_{x} u_{x} d x\right) d t \\
= & \frac{\sigma^{2}}{2} \int_{t^{\prime}}^{\infty} G\left(t-t^{\prime}, b(t), x^{\prime}\right) u_{x}(t, b(t)) d t,
\end{aligned}
$$

where we have used (129) and the fact that $\left.\frac{\sigma^{2}}{2} u G_{x}\right|_{0} ^{b(t)}=0$ by (125). Combining (135) and (131) we finally have

$$
\begin{aligned}
& \int_{t^{\prime}}^{\infty} \int_{0}^{b(t)} G\left(t-t^{\prime}, x, x^{\prime}\right) g(t, x) d x d t+u\left(t^{\prime}, x^{\prime}\right) \\
= & \frac{\sigma^{2}}{2} \int_{t^{\prime}}^{\infty} G\left(t-t^{\prime}, b(t), x^{\prime}\right) u_{x}(t, b(t)) d t
\end{aligned}
$$

Setting $x^{\prime}=b\left(t^{\prime}\right)$, we have by (125)

$$
\int_{t^{\prime}}^{\infty} \int_{0}^{b(t)} G\left(t-t^{\prime}, x, b\left(t^{\prime}\right)\right) g(t, x) d x d t=\frac{\sigma^{2}}{2} \int_{t^{\prime}}^{\infty} G\left(t-t^{\prime}, b(t), b\left(t^{\prime}\right)\right) u_{x}(t, b(t)) d t .
$$

This is the equation for $u_{x}^{I}$ at the boundary we were looking for. We show now that $u_{x}^{I I}$ solves this equation if our basic integral equation (25) has a solution. We have

$$
w_{x}^{I I}(t, b(t))=e^{-\beta t}(1-\gamma D(t)),
$$

so by $(120)$

$$
v_{x}(t, b(t)) e^{-\frac{\mu}{\sigma^{2}} b(t)+\frac{\mu^{2}}{2 \sigma^{2}} t}-\frac{\mu}{\sigma^{2}} v(t, b(t)) e^{-\frac{\mu}{\sigma^{2}} b(t)+\frac{\mu^{2}}{2 \sigma^{2}} t}=e^{-\beta t}(1-\gamma D(t)) .
$$

By (123)

$$
e^{-\frac{\mu}{\sigma^{2}} b(t)+\frac{\mu^{2}}{2 \sigma^{2}} t}\left(u_{x}(t, b(t))+\rho(t)-\frac{\mu}{\sigma^{2}}(u(t, b(t))+b(t) \rho(t))\right)=e^{-\beta t}(1-\gamma D(t)) .
$$


Since $u(t, b(t))=0$, we have

$$
u_{x}(t, b(t))=e^{\frac{\mu}{\sigma^{2}} b(t)-\frac{\mu^{2}}{2 \sigma^{2}} t-\beta t}(1-\gamma D(t))+\rho(t)\left(\frac{\mu}{\sigma^{2}} b(t)-1\right) .
$$

Set

$$
\tilde{u}(t):=u_{x}(t, b(t)) .
$$

We have $\tilde{u}(t)=\tilde{u}[b(s) ; s \geq t]$. Plugging now (141) into (137) give, after a change of variable $r=t-t^{\prime}$,

$$
\int_{0}^{\infty} \int_{0}^{b\left(r+t^{\prime}\right)} G\left(r, x, b\left(t^{\prime}\right)\right) g\left(r+t^{\prime}, x\right) d x d r=\frac{\sigma^{2}}{2} \int_{0}^{\infty} G\left(r, b\left(r+t^{\prime}\right), b\left(t^{\prime}\right)\right) \tilde{u}\left(r+t^{\prime}\right) d r,
$$

which is, after changing from $t^{\prime}$ to $t$, our basic integral equation (25) for $b(t)$. This shows that the solvability of this equation gives our final smooth fit condition (vi).

For the proof of point (viii): Analogously to the end of the proof of proposition 1 the continuity of $w_{t}$ over the boundary is a consequence of the facts that $D(t)=w(t, b(t))$ is in $C^{1}([0, \infty))$ and that $w_{x}$ is continuous over the boundary. The continuity of $w_{x x}$ follows now from the smooth fitting condition (vii).

\section{Proof of proposition 3}

The proof of the first inequality is easy. Since

$$
w(t, x)=U\left(e^{-\beta t}(x+d(t))\right)
$$

in region $I I$, it follows that for $(t, x) \in I I$

$$
\begin{aligned}
w_{t}+\mu w_{x}+\frac{\sigma^{2}}{2} w_{x x}= & e^{-\gamma e^{-\beta t}(x+d(t))} e^{-\beta t}\left(-\beta x-\beta d(t)+d^{\prime}(t)+\mu\right. \\
& \left.-\gamma \frac{\sigma^{2}}{2} e^{-\beta t}\right) \\
= & \beta e^{-\gamma e^{-\beta t}(x+d(t))} e^{-\beta t}(b(t)-x) \\
\leq & 0,
\end{aligned}
$$

where we have used (116) in the second step.

The proof of the second inequality (ii) is far more complicated. We first set

$$
m(t, x)=w_{x}(t, x)+\gamma e^{-\beta t} w(t, x)-e^{-\beta t} .
$$

We know that

$$
m(t, b(t))=0,
$$

because of the continuity of $w_{x}$ and $w$ over the boundary (by proposition $2,(\mathrm{v})+(\mathrm{vi})$ ). We also have the continuity of $w_{x x}$ over the boundary (by proposition 2,(viii)). This implies

$$
m_{x}(t, b(t))=0 .
$$

Note now that $m_{t}$ and $m_{x x}$ exist and are continuous up to the boundary by the same arguments as at the end of the proof of proposition 1 . One has just to replace in the argument there the 

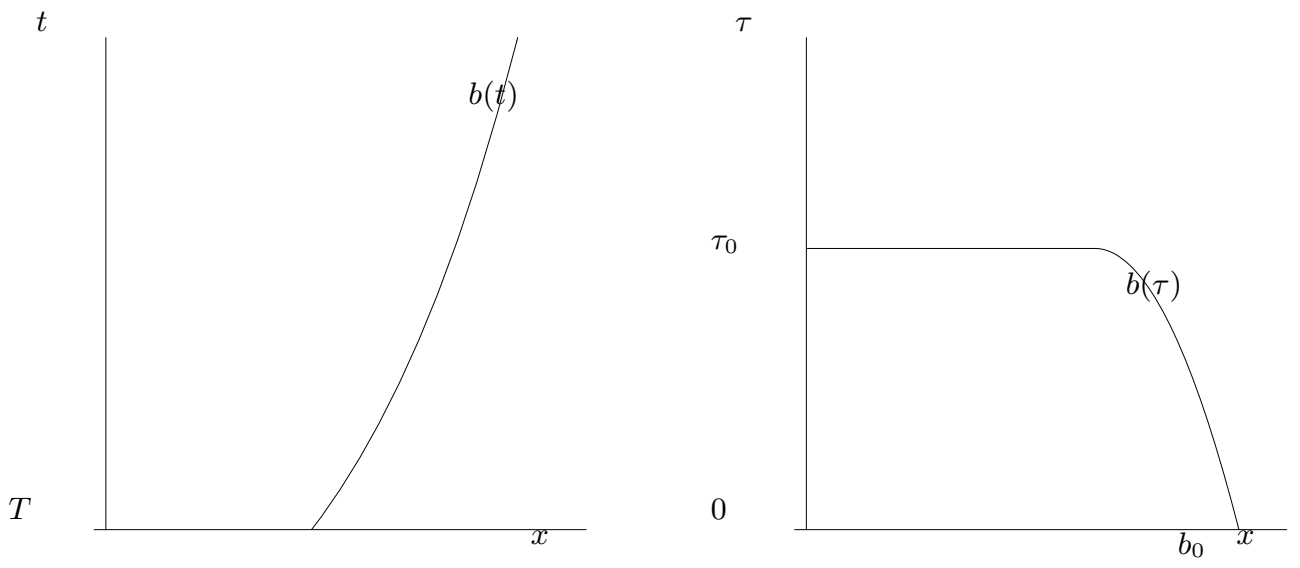

Figure 5: $b(t)$ before and after the change of coordinates.

boundary function $D(t)$ by a function identically zero, and the continuity of $w_{x}$ by the continuity of $m_{x}$. Since $m(t, b(t))=0$ we must have

$$
m_{x}(t, b(t)) b^{\prime}(t)+m_{t}(t, b(t))=0,
$$

so by $(147)$

$$
m_{t}(t, b(t))=0 \text {. }
$$

We also note that by simple calculation $m$ satisfies the following PDE

$$
m_{t}+\mu m_{x}+\frac{\sigma^{2}}{2} m_{x x}=\beta e^{-\beta t}(1-\gamma w) .
$$

Similarly as in the proof of proposition 1 we make a change of coordinates by setting

$$
\tau=e^{-t}, \quad z=\frac{x}{b(\tau)}, \quad u=w-z D(\tau) .
$$

(See figure 5.) The PDE for $u$ in new coordinates is

$$
\tau u_{\tau}=\frac{\sigma^{2}}{2 b^{2}(\tau)} u_{z z}+\frac{\mu+z \tau b^{\prime}(\tau)}{b(\tau)} u_{z}+f(\tau, z)
$$

where $f$ is defined in (107) (with $N=1$ ). We start with some easy lemmata.

Lemma $3 w(t, x)<\frac{1}{\gamma}$ in region $I$.

Proof. We use the maximum principle from Redheffer and Walter (1974). Switching to $(\tau, x)$ coordinates again, we see that we have $w(\tau, 0)=0, w(\tau, b(\tau))<\frac{1}{\gamma}$ and $w(0, x)=0$ by proposition 1. Since the functions $g$ and $f$ defined by

$$
\begin{aligned}
g\left(\tau, x, w, w_{x}, w_{\tau}\right) & =\tau w_{\tau}, \\
f\left(\tau, x, w, w_{x}, w_{x x}\right) & =\mu w_{x}+\frac{\sigma^{2}}{2} w_{x x}
\end{aligned}
$$

fulfill the conditions of Section 2 of Redheffer and Walter (1974), the maximum principle holds. 
As a consequence of lemma 3 we have

$$
m_{x x}(t, b(t))>0 .
$$

Indeed, if we had $m_{x x}(t, b(t)) \leq 0$, then the l.h.s. of $(150)$ at $(t, b(t))$ would be $\leq 0$. On the other hand, the r.h.s. of (150) is $>0$ by lemma 3 , which is a contradiction.

We continue with some more technical results.

Lemma $4 w_{t}(t, b(t)) \sim-\mu e^{-\beta t}$ for $t \rightarrow \infty$, and $w_{t}(t, b(t))<0$ for all $t \in[0, \infty)$.

Proof. By the continuity of $w_{t}$ over the boundary we have

$$
\begin{aligned}
w_{t}(t, b(t)) & =w_{t}^{I I}(t, b(t))=e^{-\beta t} e^{-\gamma e^{-\beta t}(b(t)+d(t))}\left(-\beta(b(t)+d(t))+d^{\prime}(t)\right) \\
& =(116)=e^{-\beta t} e^{-\gamma e^{-\beta t}(b(t)+d(t))}\left(-\mu+\frac{\gamma \sigma^{2}}{2} e^{-\beta t}\right) \\
& \sim-\mu e^{-\beta t} .
\end{aligned}
$$

The asserted inequality is obvious because of our assumption on $\gamma$ (see the statement of proposition 3$)$.

Lemma $5 w_{t} \leq 0$.

Proof. We consider an approximating boundary value problem in the following way. First we take a $b^{(n)}(\tau)<b(\tau)$ analytical, tending to $b(\tau)$ in the sup-norm. Then we consider

$$
\begin{array}{r}
w^{(n)}(\tau, 0)=0 \\
w^{(n)}\left(\tau, b^{(n)}(\tau)\right)=w\left(\tau, b^{(n)}(\tau)\right) \\
w^{(n)}(0, x)=0 .
\end{array}
$$

Clearly we have $w=w^{(n)}$ on $M_{n}=\left\{(\tau, x) \mid 0 \leq \tau \leq 1,0 \leq x \leq b^{(n)}\right\}$. As in the proof of proposition 1 we change to coordinates $\left(\tau, z, u^{(n)}\right)$. But now all coefficients and boundary data are smooth, so we can use Theorem 4.2 of Schuss $(1972)$ to get $u_{\tau}^{(n)}(\tau, x)$ is uniformly continuous in $x$ at $\tau=0$, implying

$$
w_{t}^{(n)}(t, x) \rightarrow 0, \quad t \rightarrow \infty
$$

We again switch to $(\tau, x)$ coordinates and calculate PDE for $K:=w_{t}^{(n)}$. Using substitution $K(\tau, x):=w_{t}(\tau, x)$ we obtain

$$
\tau K_{\tau}=\mu K_{x}+\frac{\sigma^{2}}{2} K_{x x} .
$$

We note that

$$
\begin{aligned}
K(0, x) & =w_{t}^{(n)}(0, x)=0, \\
K(\tau, 0) & =w_{t}^{(n)}(\tau, 0)=0, \\
K(\tau, b(\tau)) & =w_{t}^{(n)}\left(\tau, b^{(n)}(\tau)\right)<0
\end{aligned}
$$

for $n$ large enough by Lemma 4. Using the maximum principle of Redheffer and Walter (1974) we get $w_{t}^{(n)} \leq 0$ on $M_{n}$ and finally the assertion of the lemma by letting $n \rightarrow \infty$.

Lemma $6 w_{x} \geq 0$. 
Proof. By the maximum principle for $w$ (in $(\tau, x)$ coordinates) we have $w \geq 0$. Therefore we must have $w_{x}(\tau, 0) \geq 0$, because otherwise we would have

$$
w(\tau, \epsilon)=w(\tau, 0)+\epsilon w_{x}(\tau, 0)+o(\epsilon)<0
$$

for $\epsilon$ small, which is a contradiction to $w \geq 0$. In addition, since $w_{x}$ is continuous over the boundary we have (in $(t, x)$ coordinates)

$$
w_{x}(t, b(t))=w_{x}^{I I}(t, b(t))=e^{-\beta t} e^{-\gamma e^{-\beta t}(b(t)+d(t))},
$$

and therefore $w_{x}(\tau, b(\tau))>0$ (in $(\tau, x)$ coordinates). The PDE for $w_{x}$ in $(\tau, x)$ coordinates is

$$
\tau\left(w_{x}\right)_{\tau}-\mu\left(w_{x}\right)_{x}-\frac{\sigma^{2}}{2}\left(w_{x}\right)_{x x}=0
$$

Alltogether we have $w_{x}(\tau, 0) \geq 0, w_{x}(0, x)=0$ by proposition 1 , and $w_{x}(\tau, b(\tau))>0$. The result now follows by applying the maximum principle of Redheffer and Walter (1974).

Lemma $7 w_{x}(t, 0)$ is non-increasing in $t$.

Proof. The proof is by contradiction. Assume

$$
w_{x}\left(t_{1}, 0\right)<w_{x}\left(t_{2}, 0\right) \text { for } t_{1}<t_{2}
$$

Since

$$
\begin{aligned}
& w\left(t_{1}, \epsilon\right)=\epsilon w_{x}\left(t_{1}, 0\right)+o(\epsilon), \\
& w\left(t_{2}, \epsilon\right)=\epsilon w_{x}\left(t_{2}, 0\right)+o(\epsilon),
\end{aligned}
$$

for $\epsilon$ small enough we would have $w\left(t_{1}, \epsilon\right)<w\left(t_{2}, \epsilon\right)$, which contradicts lemma 5 .

We introduce

$$
v:=\frac{w_{x}}{1-\gamma w} .
$$

Ba a simple calculation we see that $v$ solves the PDE

$$
v_{t}+\mu v_{x}+\frac{\sigma^{2}}{2}\left(v_{x x}-2 v v_{x}\right)=0
$$

Obviously

$$
m>0 \Longleftrightarrow v>e^{-\beta t}
$$

and $v(t, 0)$ is non-increasing in $t$ by lemmas 5 and 7 .

Now we turn to Redheffer and Walter (1974) once more, paying attention to their definition of a saw (section 5, p. 63).

Lemma 8 For any $t \geq 0 v(t, \cdot)$ is a saw of maximal order 3 .

Proof. Translating (157) into $(\tau, x)$ coordinates yields

$$
\tau v_{\tau}-N \mu v_{x}+N \sigma^{2} v v_{x}-\frac{N \sigma^{2}}{2} v_{x x}=0
$$

It is easy to see that this type of PDE satisfies the assumptions of the theorem of Section 5 in Redheffer and Walter (1974). Fix $\tau^{\prime} \in[0,1]$ and observe $v$ in region $\Omega=\left\{(\tau, x) ; 0 \leq \tau \leq \tau^{\prime}, 0 \leq\right.$ $x \leq b(\tau)\}$ (see figure 6). $v(\tau, 0)$ is non-decreasing in $\tau$ because $v(t, 0)$ is non-increasing in $t$. 


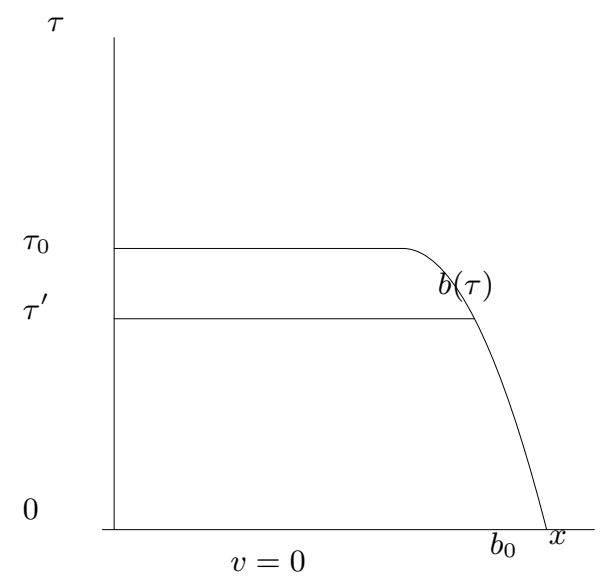

Figure 6: Region $\Omega$.

Additionally we have that $v(\tau, b(\tau))$ is non-decreasing in $\tau$ since $v(\tau, b(\tau))=\tau^{\beta}$. It follows that $v$ is a saw of order 3 on $\partial \Omega \backslash\left(\left\{\tau^{\prime}\right\} \times\left(0, b\left(\tau^{\prime}\right)\right)\right.$. Theorem of Section 5 in Redheffer and Walter (1974) now implies that $v\left(\tau^{\prime}, \cdot\right)$ is a saw of maximal order 3 .

Equation (153) together with (147) tells us that for any $t \geq 0 m(t, \cdot)$ is decreasing towards 0 in the neighborhood of $b(t)$. Therefore $v(t, \cdot)$ must be decreasing towards $e^{-\beta t}$ in the neighborhood of $b(t)$ (see figure 7 ). We now make the following assumption.

Assumption $\bar{t}$. Assume that there exists some point $\bar{t} \in[0, \infty)$ such that we do not have $m(\bar{t}, x) \geq 0 \forall x \in[0, b(t)]$. Define

$$
\bar{x}(\bar{t})=\sup \{x \in[0, b(\bar{t})) ; m(\bar{t}, x)<0\} .
$$

Analogously, we define $\bar{x}(t)$ for any $t>\bar{t}$ when it makes sense. We now have two possible cases.

Case $A . \forall t>\bar{t}$ the supremum above is non-void, i.e. there exists $\bar{x}(t)$.

Case $B$. There exists $\tilde{t}=\inf \{t>\bar{t} ; m(t, x) \geq 0 \forall x \in[0, b(t))\}$.

We want to show that neither of these cases is actually possible. Therefore the assumption above must be false. This means $m(t, x) \geq 0 \forall t \geq 0,0 \leq x \leq b(t)$, which is exactly the statement of proposition 3(ii). We will show that cases A and B are not possible in two separate lemmas.

Lemma 9 The case $A$ is not possible.

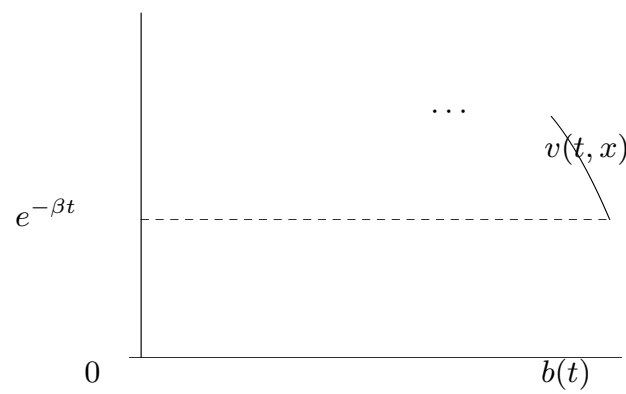

Figure 7: Behavior of $v(t, \cdot)$ at $x=b(t)$. 

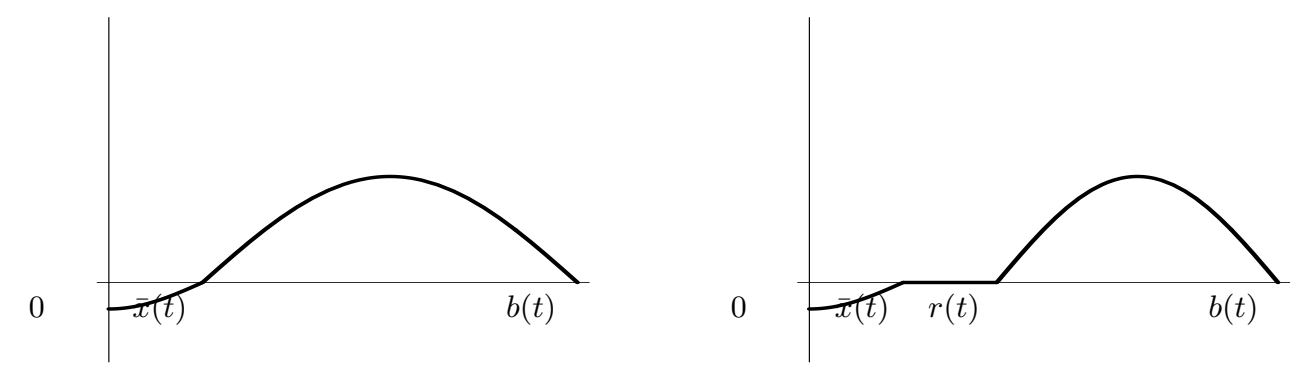

Figure 8: Two possible cases for the zeroes of $m(t, \cdot)$ : an isolated zero and an interval of zeroes.

Proof. We prove the lemma by contradiction. Assume that the statement of case A holds, and fix $t \geq 0$. Since $v(t, \cdot)$ is a saw of maximal order 3 , there are only two possibilities how $m(t, \cdot)$ may look like (see figure 8 ).

In the first case $\bar{x}(t)$ is continuous as a solution of $m(t, \bar{x}(t))=0$. In the second case, on the interval of constancy $J(t)=(\bar{x}(t), r(t))$ we have $m_{x}=m_{x x}=0$, and therefore by (150)

$$
m_{t}>0 \text { on } J(t) .
$$

This implies that $\bar{x}(t)$ is cadlag, with left limit $r(t)$.

Define for $t \geq \bar{t}$

$$
f(t):=\|m(t, \cdot)\|_{L^{1}([\bar{x}(t), b(t)])} .
$$

Since $w_{x} \geq 0$ and $w(t, b(t)) \rightarrow 0$ for $t \rightarrow \infty$

$$
\left\|w_{x}(t, \cdot)\right\|_{L^{1}([0, b(t)])} \rightarrow 0, \quad t \rightarrow \infty
$$

implying

$$
f(t)=\left\|w_{x}(t, \cdot)-e^{-\beta t}(1-\gamma w(t, \cdot))\right\|_{L^{1}([\bar{x}(t), b(t)])} \rightarrow 0, \quad t \rightarrow \infty
$$

Define

$$
M:=f(\bar{t}) .
$$

We have by (163) that there exists $t^{*}>\bar{t}$ such that

$$
f\left(t^{*}\right)<\frac{M}{2}
$$

Since $\bar{x}(t)$ is cadlag, $\left[\bar{t}, t^{*}\right)$ can be divided into a denumerable set of intervals $I_{n}$, where on each $I_{n}=\left[r_{n}, s_{n}\right) \bar{x}(t)$ is continuous.

Fix $n$ and let $t^{\prime}$ be any point in $I_{n}=\left[r_{n}, s_{n}\right)$. Choose $t>t^{\prime}$ such that $\left|t-t^{\prime}\right|$ is small and $t \in\left(r_{n}, s_{n}\right)$. We want to show

$$
f(t)-f\left(t^{\prime}\right)>0
$$

meaning that $f$ increases on $I_{n}$. We have

$$
\begin{aligned}
f(t)-f\left(t^{\prime}\right) & =\int_{\bar{x}(t)}^{\bar{x}\left(t^{\prime}\right)} m(t, x) d x+\int_{\bar{x}\left(t^{\prime}\right)}^{b(t)} m(t, x) d x-\int_{\bar{x}\left(t^{\prime}\right)}^{b\left(t^{\prime}\right)} m\left(t^{\prime}, x\right) d x \\
& =: \quad N(t)+M(t),
\end{aligned}
$$


where $N(t)$ is the first term on the r.h.s. and $M(t)$ the difference of the remaining two.

$$
\begin{aligned}
M_{t}(t)= & \int_{\bar{x}\left(t^{\prime}\right)}^{b(t)} m_{t}(t, x) d x+b^{\prime}\left(t^{\prime}\right) m(t, b(t))=(146)=\int_{\bar{x}\left(t^{\prime}\right)}^{b(t)} m_{t}(t, x) d x \\
= & \int_{\bar{x}\left(t^{\prime}\right)}^{\bar{x}(t)} m_{t}(t, x) d x \\
& +\int_{\bar{x}(t)}^{b(t)}\left(\beta e^{-\beta t}(1-\gamma w(t, x))-\mu m_{x}(t, x)-\frac{\sigma^{2}}{2} m_{x x}(t, x)\right) d x \\
= & \int_{\bar{x}\left(t^{\prime}\right)}^{\bar{x}(t)} m_{t}(t, x) d x+\int_{\bar{x}(t)}^{b(t)} \beta e^{-\beta t}(1-\gamma w(t, x)) d x \\
& -\mu(m(t, b(t))-m(t, \bar{x}(t))) \\
& -\frac{\sigma^{2}}{2}\left(m_{x}(t, b(t))-m_{x}(t, \bar{x}(t))\right) .
\end{aligned}
$$

By (146), (147) we have $m(t, b(t))=m_{x}(t, b(t))=0$. Definition of $\bar{x}(t)$ implies $m(t, \bar{x}(t))=0$, and we also have that $m_{x}(t, \bar{x}(t)) \geq 0$ holds (see figure 8 ). It follows

$$
\begin{aligned}
M_{t}(t) & \geq \int_{\bar{x}\left(t^{\prime}\right)}^{\bar{x}(t)} m_{t}(t, x) d x+\int_{\bar{x}(t)}^{b(t)} \beta e^{-\beta t}(1-\gamma w(t, x)) d x \\
& =: \quad \int_{\bar{x}\left(t^{\prime}\right)}^{\bar{x}(t)} m_{t}(t, x) d x+\eta(t) .
\end{aligned}
$$

When $t \rightarrow t^{\prime}$

$$
\int_{\bar{x}\left(t^{\prime}\right)}^{\bar{x}(t)} m_{t}(t, x) d x \rightarrow 0, \quad \eta(t) \rightarrow \eta\left(t^{\prime}\right)>0 .
$$

Consequently, for $\left|t-t^{\prime}\right|$ small enough $M_{t}(t)>\frac{1}{2} \eta\left(t^{\prime}\right)$ and since $M\left(t^{\prime}\right)=0$,

$$
M(t)=\int_{t^{\prime}}^{t} M_{t}(s) d s \geq \frac{1}{2} \eta\left(t^{\prime}\right)\left(t-t^{\prime}\right), \quad\left|t-t^{\prime}\right| \text { small enough. }
$$

For $N(t)$ we have

$$
|N(t)|=\left|\int_{\bar{x}(t)}^{\bar{x}\left(t^{\prime}\right)}\left(m(\varphi(x), x)+\int_{\varphi(x)}^{t} m_{t}(r, x) d r\right) d x\right|,
$$

where we choose $\varphi(x) \in\left[t^{\prime}, t\right]$ such that $m(\varphi(x), x)=0$. This is possible by continuity of $\bar{x}$ and taking the generalized inverse of $\bar{x}(t)$. Then

$$
|N(t)|=\left|\int_{\bar{x}(t)}^{\bar{x}\left(t^{\prime}\right)} \int_{\varphi(x)}^{t} m_{t}(r, x) d r d x\right| \leq|| m_{t} \|_{L^{\infty}\left(\left[t, t^{\prime}\right] \times\left[\bar{x}\left(t^{\prime}\right), \bar{x}(t)\right]\right)}\left(t-t^{\prime}\right)\left|\bar{x}(t)-\bar{x}\left(t^{\prime}\right)\right| .
$$

By choosing $\left|t-t^{\prime}\right|$ small enough such that

$$
\left\|m_{t}\right\|_{L^{\infty}\left(\left[t, t^{\prime}\right] \times\left[\bar{x}\left(t^{\prime}\right), \bar{x}(t)\right]\right)}\left|\bar{x}(t)-\bar{x}\left(t^{\prime}\right)\right|<\frac{1}{2} \eta\left(t^{\prime}\right)
$$


we finally get

$$
f(t)-f\left(t^{\prime}\right)>0 .
$$

Now that we have that $f(t)$ is increasing on each of the intervals $I_{n}$ we need to show that $f$ is continuous on $\left[\bar{t}, t^{*}\right]$. Set

$$
\nu(t, x)=\max (m(t, x), 0) .
$$

As $m$ is uniformly continuous on $\left\{(t, x) ; \bar{t} \leq t \leq t^{*}, 0 \leq x \leq b(t)\right\}$ the same holds for $\nu$. This implies that

$$
f(t)=\|\nu(t, \cdot)\|_{L^{1}([0, b(t)])}
$$

is clearly continuous. We conclude that $f$ is monotonously increasing on $\left[\bar{t}, t^{*}\right]$, which is a contradiction to (165). So the case A is not possible.

Lemma 10 The case $B$ is not possible.

Proof. We again prove the lemma by contradiction. Assume that the statement of case B holds. Let $\tilde{t}=\inf \{t>\bar{t} ; m(t, x) \geq 0 \forall x \in[0, b(t))\}$. By continuity of $m, \tilde{t}>\bar{t}$ must hold. At $x=0$ we have

$$
m(\tilde{t}, 0)=w_{x}(\tilde{t}, 0)-e^{-\beta \tilde{t}}(1-\gamma w(\tilde{t}, 0))=w_{x}(\tilde{t}, 0)-e^{-\beta \tilde{t}} \geq 0,
$$

so

$$
w_{x}(\tilde{t}, 0) \geq e^{-\beta \tilde{t}}
$$

Since $w_{t}(\tilde{t}, 0)=0$,

$$
w_{x x}(\tilde{t}, 0)=-\frac{2 \mu}{\sigma^{2}} w_{x}(\tilde{t}, 0)<0 .
$$

Then

$$
m_{x}(\tilde{t}, 0)=w_{x x}(\tilde{t}, 0)+\gamma e^{-\beta \tilde{t}} w_{x}(\tilde{t}, 0)=w_{x x}(\tilde{t}, 0)\left(1-\frac{\gamma \sigma^{2}}{2 \mu} e^{-\beta \tilde{t}}\right),
$$

implying

$$
m_{x}(\tilde{t}, 0)<0 \quad \text { since } \quad \gamma<\frac{2 \mu}{\sigma^{2}} .
$$

On the other hand by uniform continuity of $m$ on $\{(t, x) ; \bar{t} \leq t \leq \tilde{t}, 0 \leq x \leq b(t)\}$ there has to be a point $\tilde{x} \in[0, b(t)]$ such that

$$
m(\tilde{t}, \tilde{x})=0 .
$$

Otherwise we would have $m(\tilde{t}-\epsilon, x)>0 \forall x \in[0, b(\tilde{t}-\epsilon)]$ for some $\epsilon>0$, contradicting the definition of $\tilde{t}$. $\tilde{x} \neq 0$ by (174) and $\tilde{x} \neq b(\tilde{t})$ by (153), so

$$
\tilde{x} \in(0, b(\tilde{t})) .
$$

In that case $m(\tilde{t}, \cdot)$ and $v(\tilde{t}, \cdot)$ can only look like in figure 9 . We see that in this case $v(\tilde{t}, x)$ is a saw of order at least 4 , which is a contradiction to lemma (8). So the case B is not possible.

Acknowledgements: Support by the "Christian Doppler Gesellschaft" , Project PRISMA, is gratefully acknowledged.

We also thank an anonymous referee for suggestions to improve the paper. 

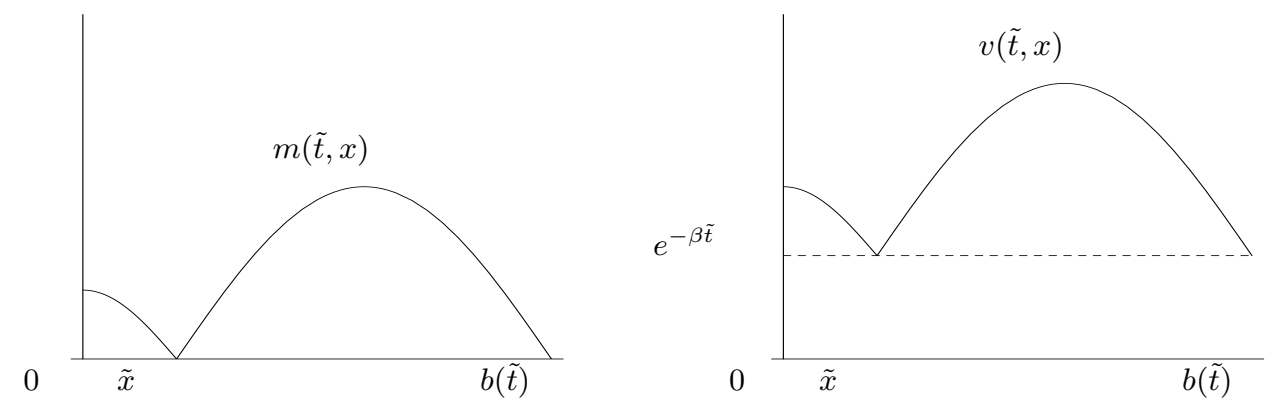

Figure 9: Behavior of $m(\tilde{t}, \cdot)$ and $v(\tilde{t}, \cdot)$.

\section{References}

Albrecher, H. and R. Kainhofer (2002). Risk theory with a nonlinear dividend barrier. Computing 68(4), 289-311.

Amann, H. (1993). Nonhomogeneous linear and quasilinear elliptic and parabolic boundary value problems. In Function spaces, differential operators and nonlinear analysis, pp. 9-126. Stuttgart: Teubner.

Asmussen, S. and M. Taksar (1997). Controlled diffusion models for optimal dividend pay-out. Insurance Math. Econom. 20(1), 1-15.

Buttu, A. (1992). On the evolution operator for a class of nonautonomous abstract parabolic equations. J. Math. Anal. Appl. 170(1), 115-137.

Da Prato, G. and P. Grisvard (1979). Equations d'évolution abstraites non linéaires de type parabolique. Ann. Mat. Pura Appl. (4) 120, 329-396.

Fleming, W. H. and H. M. Soner (1993). Controlled Markov processes and viscosity solutions. New York: Springer.

Friedman, A. and Z. Schuss (1971). Degenerate evolution equations in Hilbert space. Trans. Amer. Math. Soc. 161, 401-427.

Gaier, J., P. Grandits, and W. Schachermayer (2003). Asymptotic ruin probabilities and optimal investment. Ann. Appl. Probab. 13(3), 1054-1076.

Gerber, H. (1969). Entscheidungskriterien für den zusammengesetzten Poisson-Prozess. Schweiz. Verein. Versicherungsmath. Mitt. 69, 185-228.

Gerber, H. U. (1981). On the probability of ruin in the presence of a linear dividend barrier. Scand. Actuar. J. (2), 105-115.

Gerber, H. U. and E. S. W. Shiu (2004). Optimal dividends: analysis with Brownian motion. $N$. Am. Actuar. J. 8(1), 1-20.

Grandits, P. (2004). An analogue of the Cramér-Lundberg approximation in the optimal investment case. Appl. Math. Optim. 50(1), 1-20.

Hipp, C. and M. Plum (2000). Optimal investment for insurers. Insurance Math. Econom. 27(2), $215-228$. 
Hubalek, F. and W. Schachermayer (2004). Optimizing expected utility of dividend payments for a Brownian risk process and a peculiar nonlinear ODE. Insurance Math. Econom. 34(2), $193-225$.

Kalashnikov, V. and R. Norberg (2002). Power tailed ruin probabilities in the presence of risky investments. Stochastic Process. Appl. 98(2), 211-228.

Lions, J.-L. and E. Magenes (1972). Non-homogeneous boundary value problems and applications. Berlin: Springer.

Paulsen, J. (2002). On Cramér-like asymptotics for risk processes with stochastic return on investments. Ann. Appl. Probab. 12(4), 1247-1260.

Redheffer, R. M. and W. Walter (1974). The total variation of solutions of parabolic differential equations and a maximum principle in unbounded domains. Math. Ann. 209, 57-67.

Renardy, M. and R. C. Rogers (1993). An introduction to partial differential equations. New York: Springer.

Schmidli, H. (2005). On optimal investment and subexponential claims. Insurance Math. Econom. 36(1), 25-35.

Schuss, Z. (1972). Regularity theorems for solutions of a degenerate evolution equation. Arch. Rational. Mech. Anal. 46, 200-211.

Shreve, S., J. Lehocsky, and D. Gaver (1984). Optimal consumption for general diffusions with absorbing and reflecting barriers. SIAM J. Control and Optimization 22, 55-75.

Address of the corresponding author:

Peter Grandits

Institute for Mathematical Methods in Economics (E105)

TU Vienna

Wiedner Haupstr. 8-10

1040 Vienna

Austria

pgrand@fam.tuwien.ac.at 